\title{
Topological analysis of the quantum Hall effect in graphene: Dirac-Fermi transition across van Hove singularities and edge versus bulk quantum numbers
}

\author{
Yasuhiro Hatsugai \\ Department of Applied Physics, University of Tokyo, Hongo, Tokyo 113-8656, Japan \\ Takahiro Fukui \\ Department of Mathematical Sciences, Ibaraki University, Mito 310-8512, Japan \\ Hideo Aoki \\ Department of Physics, University of Tokyo, Hongo, Tokyo 113-0033, Japan
}

(Received 21 July 2006; published 13 November 2006)

\begin{abstract}
Inspired by a recent discovery of a peculiar integer quantum Hall effect (QHE) in graphene, we study QHE on a honeycomb lattice in terms of the topological quantum number, with two interests. First, how the zero-mass Dirac QHE around the center of the tight-binding band crosses over to the ordinary finite-mass fermion QHE around the band edges. Second, how the bulk QHE is related with the edge QHE for the entire spectrum including Dirac and ordinary behaviors. We find the following. (i) The zero-mass Dirac QHE [with $\sigma_{x y}=\mp(2 N+1) e^{2} / h, N$ : integer] persists, surprisingly, up to the van Hove singularities, at which the ordinary fermion behavior abruptly takes over. Here a technique developed in the lattice gauge theory enabled us to calculate the behavior of the topological number over the entire spectrum. This result indicates a robustness of the topological quantum number, and should be observable if the chemical potential can be varied over a wide range in graphene. (ii) To see if the honeycomb lattice is singular in producing the anomalous QHE, we have systematically surveyed over square $\leftrightarrow$ honeycomb $\leftrightarrow \pi$-flux lattices, which is scanned by introducing a diagonal transfer $t^{\prime}$. We find that the massless Dirac QHE $[\propto(2 N+1)]$ forms a critical line, that is, the presence of Dirac cones in the Brillouin zone is preserved by the inclusion of $t^{\prime}$ and the Dirac region sits side by side with ordinary one persists all through the transformation. (iii) We have compared the bulk QHE number obtained by an adiabatic continuity of the Chern number across the square $\leftrightarrow$ honeycomb $\leftrightarrow \pi$-flux transformation and numerically obtained edge QHE number calculated from the whole energy spectra for sample with edges, which shows that the bulk QHE number coincides, as in ordinary lattices, with the edge QHE number throughout the lattice transformation.
\end{abstract}

DOI: 10.1103/PhysRevB.74.205414

PACS number(s): 73.43. $-\mathrm{f}$

\section{INTRODUCTION}

Electrons on a honeycomb lattice, despite its simplicity, provide interesting problems in condensed matter physics, especially in its topological aspects. Field theoretically, Dirac particles and associated gauge fields have been intensively investigated from a topological point of view, ${ }^{1,2}$ so electronic properties for the honeycomb lattice may open new avenues for condensed matter phenomena. Indeed, there have been several proposals about peculiar properties in condensed matter systems that have zero-mass Dirac particles at low energy scales. ${ }^{3,4}$ Apart from honeycomb lattice, zero-mass Dirac particles appear in condensed matter physics as effective theories in various guise. These include the $d$-wave superconductivity, 5,6 the so-called $\pi$-flux or chiral spin state, ${ }^{7,8}$ Anderson localization problems, ${ }^{5,9}$ spin related problems on the honeycomb ${ }^{11}$ or Kagome ${ }^{10}$ lattice, and quantum phase transitions in two dimensions. ${ }^{12,13}$

A seminal highlight, however, is the anomalous quantum Hall effect (QHE) in the honeycomb lattice, ${ }^{14-18}$ which has recently been observed ${ }^{19,20}$ in graphene, a monolayer graphite with a honeycomb array of carbon atoms. By the anomalous quantum Hall effect is meant the sequence $\sigma_{x y}=\mp(2 N$ $+1) e^{2} / h$, ( $N$ : integer) for the quantum Hall steps per spin 15,16 . While the study of graphite has a long history, recent advances have been directed toward nanostructured graphite such as the carbon nanotube ${ }^{21}$ or nanographite with boundary magnetism expected to arise from edge states. ${ }^{22,23}$ In this context, the monolayer graphene is particularly interesting as an ideal realization of the honeycomb lattice, and the discovery of QHE has kicked off intensive studies.

Quite generally, topologically nontrivial states are characterized not by local order parameters as in symmetry breaking states, but by geometrical phases, ${ }^{24,25}$ where what are now known as topological orders can be realized. ${ }^{26-29}$ One interesting consequence is that topological quantum numbers for the bulk can often be related with those for the edge states in finite systems. With this bulk-edge correspondence topological properties which can be hidden in the bulk may thus become visible around the boundaries. A typical example is the edge states in QHE systems, which reflect a nontrivial topological structure of the bulk. ${ }^{30-33}$

For the QHE on the honeycomb lattice, we can then pose two fundamental questions. (i) While the low-energy theory around the band center $(E=0)$ is that of the zero-mass Dirac particle, which is now realized to give an anomalous QHE, how this should be taken over by ordinary theories as we go away from the band center? (ii) How should the bulk-edge correspondence look for the zero-mass Dirac particle? The question (ii) is of special interest in the context of the zero 
mode of the massless Dirac particles. ${ }^{34,35}$ There, a bipartite structure (chiral symmetry) in the honeycomb lattice is intimately related to the appearance of zero mode edge states. 36,39

As for the question (i), Zheng and Ando ${ }^{15}$ have numerically calculated the QHE on honeycomb with a selfconsistent Born approximation. They have calculated not only the anomalous QHE around $E=0$ but also the Hall conductivity for the entire energy region with this approximation. More recently, Sheng et al. ${ }^{40}$ have examined the QHE in graphene, computing the QHE number over the whole energy spectrum. They have shown that Dirac-like quantization of the Hall conductivity appears only near the zero energy and conventional quantization can be observed in the band edge region. However, our question here is on the precise topological quantum number ${ }^{41}$ in the region including around the boundary between the anomalous and ordinary ones.

So in this paper, we explore the topological aspects for electrons on the honeycomb lattice in a magnetic field. We show the following.

(i) The zero-mass Dirac particle behavior [with the Hall conductivity $\sigma_{x y}= \pm(2 N+1) e^{2} / h$, where $N$ is an integer and we ignore spin degeneracy] persists, surprisingly, up to finite energies, at which the usual finite-mass fermion behavior abruptly takes over. The boundary energies are identified to be the van Hove singularities. Here a technique developed in the lattice gauge theory enabled us to calculate the behavior of the topological number, which can become huge over the entire spectrum. This result indicates a robustness of the topological quantum number, and should be observable if the chemical potential can be varied over a wide range in graphene.

(ii) To see if the honeycomb lattice is singular in producing the anomalous QHE, we have systematically surveyed the systems that extend over square $\leftrightarrow$ honeycomb $\leftrightarrow \pi$-flux lattices by introducing a diagonal transfer $t^{\prime}$. We find that the Dirac region always exists (and sitting side by side with ordinary one with it's boundary dependent on $t^{\prime}$ ) all through the transformation (except at the square lattice, which, with merged van Hove singularities, is rather singular in the present viewpoint). It implies the massless Dirac fermion forms a critical line in a parameter space. It does not occur as a critical point by adjusting parameters. Incidentally, we make an interesting observation, in the region honeycomb $\leftrightarrow \pi$-flux lattices, that the presence of multiple extrema in the band dispersion can give rise to a fermion doubling with the Chern number $\propto 2 N$ rather than $(2 N+1){ }^{42}$

(iii) We have then compared (a) the bulk QHE number obtained by an adiabatic continuity of the Chern number across the square $\leftrightarrow$ honeycomb $\leftrightarrow \pi$-flux transformation and (b) numerically obtained edge QHE number calculated from the whole energy spectra for sample with edges. The result shows that the bulk QHE number coincides, as in ordinary lattices, with the edge QHE number throughout the lattice transformation. Incidentally, the $E=0$ flat edge mode persists in strong magnetic fields.

The organization of the paper is as follows. In the next section, we define the tight-binding model on the honeycomb lattice, where we introduce a diagonal transfer to tune the position of the van Hove singularities to go over to the square and $\pi$-flux lattices. We present numerical results for the energy spectra as a function of a magnetic field (Hofstadter diagram), which enables us to infer a topological relationship among the honeycomb, square, and $\pi$-flux lattices. In Sec. III, we compute the Hall conductivity of the bulk as a function of the chemical potential, based on a lattice-gauge theoretical method developed in Ref. 47. This calculation is manifestly gauge invariant and guarantees integer Chern numbers, so that the method is powerful in evaluating the QHE topological number over the whole spectrum, including the van Hove singularities, which turn out to accompany singular behaviors in QHE. There the topological equivalence in Sec. II B is thus confirmed with respect to the bulk topological properties. In Sec. IV, we show that the conversion square $\leftrightarrow$ honeycomb $\leftrightarrow \pi$-flux has a virtue of enabling us to derive the Diophantine equation for the Dirac-fermion regime from the adiabatic principle for the topological quantum number, which exists as far as the energy gap in which $E_{F}$ reside does not close. Section $\mathrm{V}$ is devoted to the edge states of the model. Edge states in finite (cylindrical) systems are analyzed with the transfer matrix formalism, and we show that edge states, with Laughlin's argument, ${ }^{30}$ indeed reveal the Dirac-like quantization sitting next to the conventional quantization separated by van Hove singularities. Thus, the bulk-edge correspondence is confirmed. A summary and discussion is given in Sec. VI.

\section{MODEL AND TOPOLOGICAL EQUIVALENCE}

Let us start with defining the Hamiltonian for a model that interpolates honeycomb lattice with square and $\pi$-flux lattices. This parameter plays a key role in examining the topological properties in terms of the adiabatic principle.

\section{A. Model}

The Hamiltonian for two-dimensional tight-binding systems in a uniform magnetic field $\boldsymbol{B}$ applied normal to the plane is

$$
H=H_{\text {honeycomb }}+H^{\prime} \text {. }
$$

Here, $H_{\text {honeycomb }}$ is the tight-binding model on the honeycomb lattice with nearest-neighbor hopping

$$
\begin{aligned}
H_{\text {honeycomb }}= & t \sum_{j}\left[c_{\bullet}^{\dagger}(\mathbf{j}) c_{\circ}(\mathbf{j})+e^{i 2 \pi \phi j_{1}} c_{\bullet}^{\dagger}(\mathbf{j}) c_{\circ}\left(\mathbf{j}-\mathbf{e}_{2}\right)\right. \\
& \left.+c_{\bullet}^{\dagger}\left(\mathbf{j}+\mathbf{e}_{1}\right) c_{\circ}(\mathbf{j})\right]+ \text { H.c. },
\end{aligned}
$$

where $t=-1$ is the transfer (taken to be the unit of energy). Since the honeycomb, a non-Bravais lattice with two sites per unit cell is bipartite, we have defined two kinds of fermion operators, $c_{\circ}(j)$ and $c_{0}(j)$ as in Fig. 1 , where $\mathbf{j}=j_{1} \mathbf{e}_{1}$ $+j_{2} \mathbf{e}_{2}$ labels the unit cell with two translation vectors $e_{1}$ $=(3 / 2, \sqrt{3} / 2) a$ and $\mathbf{e}_{2}=(0, \sqrt{3}) a$. The magnetic field is characterized by

$$
\phi=B S_{6} /(2 \pi) \equiv p / q,
$$

the magnetic flux (assumed to be rational) penetrating each hexagon of area $S_{6}=(3 \sqrt{3} / 2) a^{2}$. We have adopted the Landau 


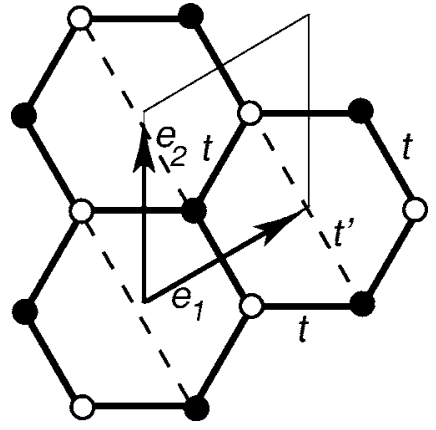

FIG. 1. A honeycomb lattice with a unit cell and a extra transfer $t^{\prime}$ indicated.

gauge for the vector potential. For simplicity we neglect the spin degrees of freedom, since the graphene has a very small Zeeman splitting. If we add a hopping across each hexagon,

$$
H^{\prime}=t^{\prime} \sum_{\mathbf{j}} e^{-i 2 \pi \phi\left(j_{1}+1 / 2\right)} c_{\bullet}^{\dagger}\left(\mathbf{j}+\mathbf{e}_{1}-\mathbf{e}_{2}\right) c_{\circ}(j)+\text { H.c. },
$$

we can change the system continuously from $t^{\prime} / t=-1$ (which is referred to as $\pi$-flux lattice) to 0 (honeycomb) and 1 (square).

In the momentum space, the Hamiltonian for $\phi=p / q$ is expressed as

$$
H=\int_{0}^{2 \pi / q} \frac{d k_{1}}{2 \pi / q} \int_{0}^{2 \pi} \frac{d k_{2}}{2 \pi} \boldsymbol{c}^{\dagger}(\boldsymbol{k}) h(\boldsymbol{k}) \boldsymbol{c}(\boldsymbol{k}),
$$

where $\boldsymbol{c}^{\dagger}(\boldsymbol{k})$ is a $2 q$ dimensional vector and $h(\boldsymbol{k})$ is a $2 q$ $\times 2 q$ matrix (see Appendix A). In zero magnetic field, we have

$$
\begin{aligned}
h(\boldsymbol{k})= & t\left(\begin{array}{ll}
0 & \Delta(\boldsymbol{k}) \\
\Delta^{*}(\boldsymbol{k}) & 0
\end{array}\right), \Delta(\boldsymbol{k})=1+e^{-i k_{2}}+e^{-i k_{1}}[1 \\
& \left.+\left(t^{\prime} / t\right) e^{+i k_{2}}\right] .
\end{aligned}
$$

To see how $t^{\prime}$ shifts the position of van Hove singularities, we show the energy dispersions and the density of states in the zero magnetic field in Figs. 2 and 3. The square lattice has a van Hove singularity at the band center, which splits into two as we decrease $t^{\prime} / t$ from 1 . As we shall stress, it is between the two singularities that a zero-mass Dirac-like gapless dispersion appears in the honeycomb lattice. We can in fact rigorously show ${ }^{37}$ that the zero gap with a linearly vanishing density of states around $E=0$ is not an accident for honeycomb, but exists for $-3<t^{\prime}<1$, so such a situation persists down to the $\pi$-flux lattice.

Ordinary band dispersions and density of states reside outside the van Hove singularities. So our question here is how the quantum Hall effect should look along the sequence, square $\leftrightarrow$ honeycomb $\leftrightarrow \pi$-flux.

\section{B. Topological equivalences}

In a magnetic field $B$, the spectra of the lattice Hamiltonians against $B \propto \phi$ take fractal shapes, usually called Hofstadter's diagram, ${ }^{38}$ where hierarchical series of energy gaps exist. The butterfly is deformed as $t^{\prime}$ is varied. Here, an adiabatic principle plays a crucial role: it dictates that one can keep track of quantum mechanical ground states when the Hamiltonian is transformed with a continuous change of parameter(s) if there is a gap above the ground state and if the gap remains finite throughout. ${ }^{13}$ Let us apply this argument to the present model, where $t^{\prime}$ is the adiabatic parameter. In Hofstadter's butterfly we can see many Landau bands. The change of $t^{\prime}$ makes some of the bands merge (or, more precisely, some of the gaps between Landau bands merge, since the spectrum is fractal). The Landau levels are characterized by the quantum Hall numbers, which are topological (Chern) numbers as will be discussed in Sec. III, and the numbers remain unchanged against the adiabatic change as long as the gap in which $E_{F}$ reside does not collapse. This is a topological stability.

\section{Topological equivalences between van-Hove singularities}

The Hofstadter diagram for honeycomb lattice was first obtained in Ref. 43. Rammal has already noted the presence of the $E=0$ Landau level which is outside Onsager's semiclassical quantization scheme. The spectrum for the honeycomb lattice is symmetric about $\phi=\pi$. In Figs. 4, we show how the spectrum versus $\phi \propto B$ is deformed with $t^{\prime}$ for the square lattice $\left(t^{\prime} / t \rightarrow 1\right)$, or for the $\pi$-flux lattice $\left(t^{\prime} / t \rightarrow-1\right)$. In the present construction the flux per plaquette on the square lattice corresponds to half the hexagon in the honeycomb lattice, which is why Hofstadter's diagram has a period of $4 \pi$ for $t^{\prime} \neq 0$. The property that the spectrum is invariant against $\phi \rightarrow-\phi$ as well as against $t^{\prime} \leftrightarrow-t^{\prime}, \phi \leftrightarrow 1-\phi$ is retained throughout. The honeycomb lattice with $t^{\prime}=0$ corresponds to the self-dual point for the $t^{\prime} \leftrightarrow-t^{\prime}$ symmetry. (a)

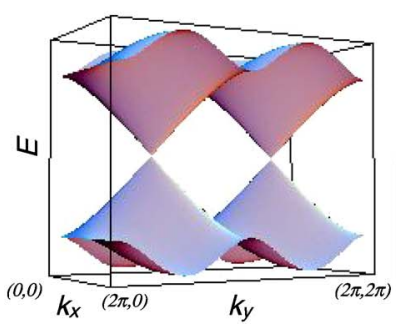

(b) $\quad t^{\prime} / t=-0.5$

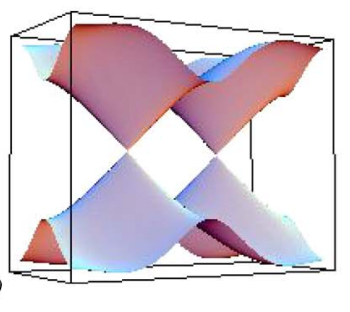

(c) honeycomb: $t^{\prime} / t=0$

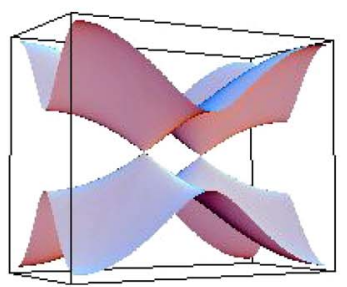

(d) $\quad t^{\prime} / t=+0.5$

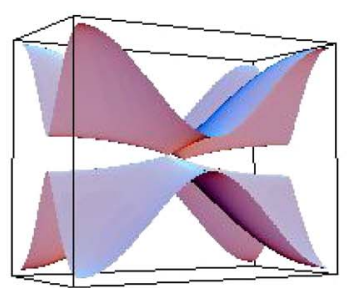

(e) square : $t^{\prime} / t=+1$

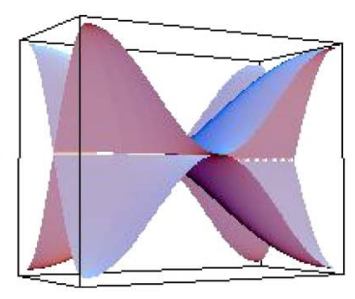

FIG. 2. (Color online) Energy dispersions for (a) $t^{\prime} / t=-1$ ( $\pi$-flux lattice), (b) $t^{\prime} / t=0.5$, (c) $t^{\prime} / t=0$ (honeycomb), (d) $t^{\prime} / t=0.5$, and (e) $t^{\prime} / t=1$ (square). 


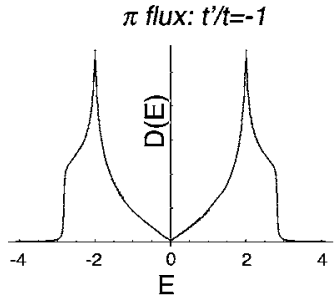

(a)

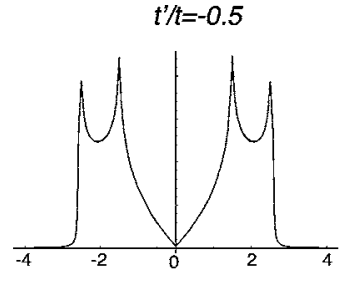

(b)

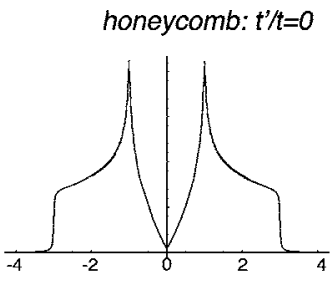

(c)

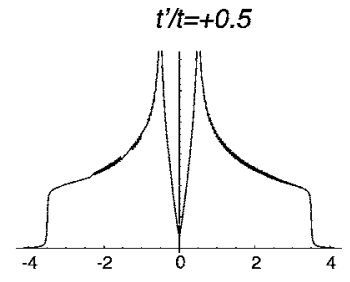

(d)

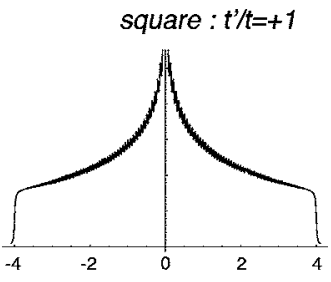

(e)

FIG. 3. Density of states for (a) $t^{\prime} / t=-1$ ( $\pi$-flux lattice), (b) $t^{\prime} / t=0.5$, (c) $t^{\prime} / t=0$ (honeycomb), (d) $t^{\prime} / t=0.5$, and (e) $t^{\prime} / t=1$ (square).

We can immediately notice from Figs. 4(a) and 4(b) that the large gaps just above and below the zero energy remain. This implies that the topological number should remain the same when $E_{F}$ lies in the gap. A closer examination shows, surprisingly, that this holds for other gaps, all the way up to a finite energy $E_{c}$, in fact. Figure 5 is a blowup of the low magnetic field region. We can see that there is no level crossing for other energy gaps as well in the energy region $E$ $\leq E_{c}$, so that we can keep track of the main gaps in the adiabatic change between the honeycomb and $\pi$ flux $\left(t^{\prime} / t\right.$ $=0 \leftrightarrow-1)$. The boundary energy $E_{c}$ is seen to reside around the region where the Landau level fan extending from $E=0$ meets another Landau fan extending from the band edges $|E| \simeq 3$. We shall show below, by using the calculation of the quantum Hall numbers, that we can identify $E_{c}$ as the van Hove energies (which are functions of $t^{\prime}$ ).

\section{Topological equivalences near band edges}

The above situation is exactly symmetric for the crossover of the honeycomb $\leftrightarrow$ square $\left(t^{\prime} / t=0 \leftrightarrow 1\right)$, where we have only to replace $\phi$ with $1-\phi$. This means that, in the weak magnetic field region depicted in Fig. 5, large gaps around $E=0$ are closed as we go to the square lattice, while we can keep track of the Landau fan starting from the band edge. This property is in fact highly nontrivial, since the Landau levels is usually analyzed in the effective-mass approach only near the band center or band edges. This is exactly why we want to confirm the topological equivalences in terms of the quantum Hall number in the next section.

\section{TOPOLOGICAL EQUIVALENCES IN THE BULK}

\section{A. Hall condactance}

It is now firmly established that the Hall conductivity of the noninteracting $2 \mathrm{D}$ electron systems, as described with the linear response theory, ${ }^{46}$ may be regarded as a topological quantum number. ${ }^{44,45}$ Namely, when the Fermi energy lies in the $n$th gap in a general two-dimensional periodic system, the Hall conductivity is given by

$$
\sigma_{x y}=-\frac{e^{2}}{h} c_{F}\left(E_{F}\right),
$$

where $c_{F}$ is an integer called Chern number, which describes how the wave function (a vector bundle) responds to a vector potential generated by Berry's gauge potential in the Brillouin zone. To compute the number, we need to diagonalize the Hamiltonian

$$
h(\boldsymbol{k}) \boldsymbol{\varphi}_{j}(\boldsymbol{k})=\boldsymbol{\epsilon}_{j}(\boldsymbol{k}) \boldsymbol{\varphi}_{j}(\boldsymbol{k})(j=1,2, \ldots, 2 q),
$$

where $h(\boldsymbol{k})$ is the $2 q \times 2 q$ matrix defined in Eq. (A1), $\boldsymbol{\varphi}_{j}(\boldsymbol{k})$ an eigenvector, and we assume that $\epsilon_{1}(k) \leq \cdots \leq \epsilon_{n}(k)<E_{F}$ $<\epsilon_{n+1}(k) \leq \cdots \leq \epsilon_{2 q}(k)$ holds over the entire Brillouin zone. We can then define Berry's gauge potential

$$
(\boldsymbol{A}(\boldsymbol{k}))_{i j}=\boldsymbol{\varphi}_{i}^{\dagger}(\boldsymbol{k}) \boldsymbol{\nabla}_{\boldsymbol{k}} \boldsymbol{\varphi}_{j}(\boldsymbol{k})(1 \leq i, j \leq n),
$$

where $\boldsymbol{A}(\boldsymbol{k})$ is an anti-Hermitian $n \times n$ matrix. The Chern number is given as the $\mathrm{U}(1)$ part of the above $\mathrm{U}(n)$ gauge potential

$$
c_{F}\left(E_{F}\right)=\frac{1}{2 \pi i} \int \operatorname{Tr} d A,
$$

where $A(\boldsymbol{k}) \equiv A_{\mu}(\boldsymbol{k}) d k_{\mu}$ is a one-form.

This formulation, a non-Abelian extension of Berry's gauge potential, ${ }^{28,29}$ may seem too elaborate, but is useful when there are multiple bands below the Fermi energy. Namely, this formula holds even if some gaps in the Fermi sea are closed, as long as the bands in question $\epsilon_{n}(\boldsymbol{k})$ and $\epsilon_{n+1}(\boldsymbol{k})$, do not cross. In the special case when all the bands in the Fermi sea are separated with each other, the Chern number, (3) is simply the sum of the Chern numbers assigned to individual bands, ${ }^{45}$ but we have opted for the above formula, since some of the gaps do collapse as $t^{\prime}$ is varied, as we shall see.

\section{B. Lattice gauge theory technique}

To actually evaluate the topological integer for the present system, we need to calculate eigenfunctions of the $2 q \times 2 q$ Hamiltonian (A1). Diagonalization can be done only numerically, so that the eigenfunctions are obtained in practice on mesh points in the Brillouin zone. This can cause a serious problem if we want to obtain the Chern numbers, especially around the van Hove singularities, since they can behave wildly there. The problem becomes even more formidable if we want to maintain the calculation manifestly gauge invariant and pin point the Chern numbers as integers. Here we adopt a method, ${ }^{47,48}$ developed recently in the context of the lattice gauge theory. ${ }^{49-54}$ This implementation precisely guarantees manifest gauge invariance and integer Chern numbers.

Let us first compute the eigenfunction of the Hamiltonian on meshes in the Brillouin zone. In what follows, we choose the Landau gauge for the magnetic field, so that the mesh points are denoted as $\left(k_{j_{1}}, k_{j_{2}}\right)$, where discrete sets of mo- 

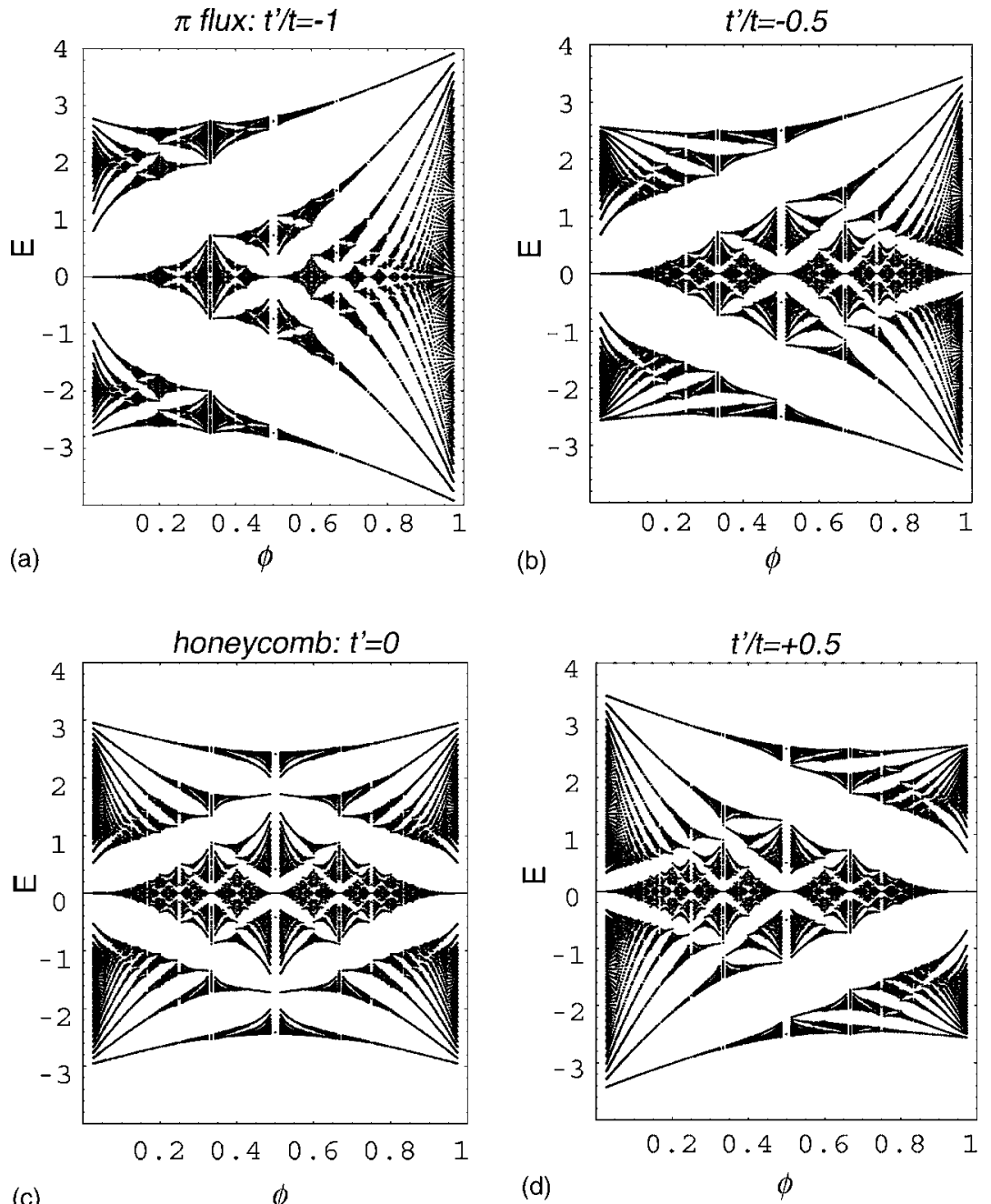

FIG. 4. Hofstadter's diagram (energy spectrum against the magnetic flux $\phi$ ) for (a) $t^{\prime} / t=-1$ ( $\pi$-flux lattice), (b) $t^{\prime} / t=0.5$, (c) $t^{\prime} / t=0 \quad$ (honeycomb), (d) $t^{\prime} / t$ $=0.5$, and (e) $t^{\prime} / t=1$ (square).

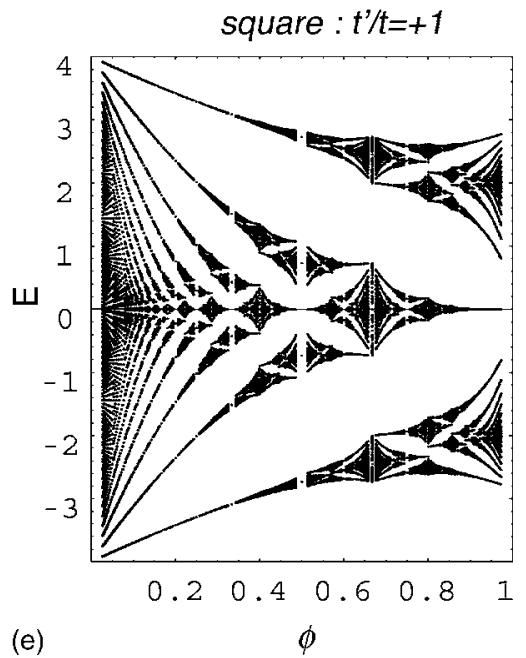

(e)

menta with $j_{\mu}=0, \ldots, N_{\mu}-1$ are defined by $k_{j_{1}}=2 \pi j_{1} /\left(q N_{1}\right)$ and $k_{j_{2}}=2 \pi j_{2} / N_{2}$ in the Brillouin zone extending over 0 $\leq k_{1}<2 \pi / q$ and $0 \leq k_{2}<2 \pi$. Diagonalization of $h(\boldsymbol{k})$ on these sites $\boldsymbol{k}=\boldsymbol{k}_{l} \equiv\left(k_{j_{1}}, k_{j_{2}}\right), l=1, \ldots, N_{1} N_{2}$ provides eigenfunctions with $h\left(\boldsymbol{k}_{l}\right) \boldsymbol{\varphi}_{j}\left(\boldsymbol{k}_{l}\right)=\boldsymbol{\epsilon}_{j}\left(\boldsymbol{k}_{l}\right) \boldsymbol{\varphi}_{j}\left(\boldsymbol{k}_{l}\right)$, which can be chosen to satisfy the periodic boundary condition $\boldsymbol{\varphi}_{j}\left(\boldsymbol{k}_{l}+N_{\mu} \hat{\boldsymbol{\mu}}\right)$ $=\boldsymbol{\varphi}_{j}\left(\boldsymbol{k}_{l}\right)$, where $\hat{\boldsymbol{\mu}}$ is a vector of length $|\hat{\mathbf{1}}|=2 \pi /\left(q N_{1}\right)$ and $|\hat{\mathbf{2}}|=2 \pi / N_{2}$ along $\boldsymbol{k}_{\mu}$.

Provided that the Fermi energy lies between the $n$th gap [namely, $\boldsymbol{\epsilon}_{n}\left(\boldsymbol{k}_{l}\right)<E_{F}<\boldsymbol{\epsilon}_{n+1}\left(\boldsymbol{k}_{l}\right)$ for every $\boldsymbol{k}_{l}$, and hence the Fermi sea is composed of $n$ bands (some of which may merge), we define a U(1) link variable of the Fermi sea as 


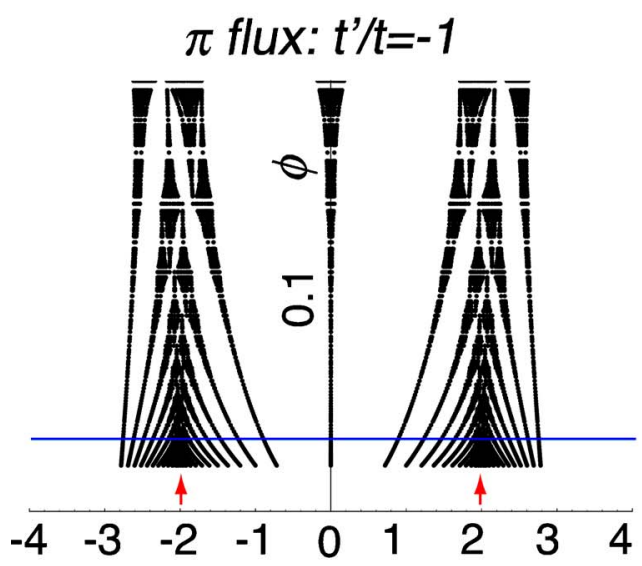

(a)

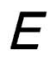

honeycomb: $t^{\prime} / t=0$

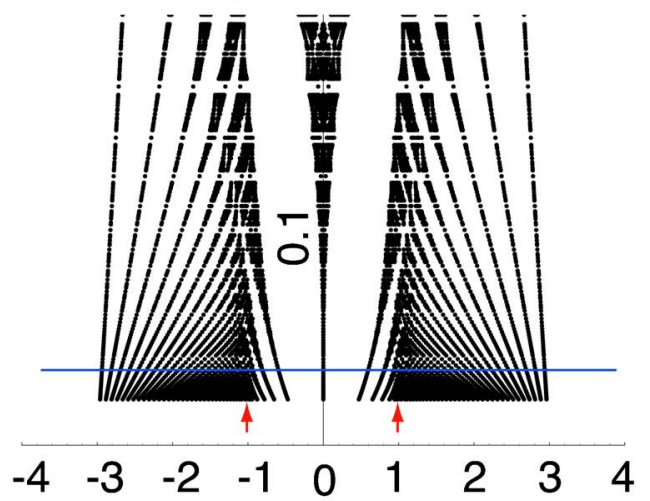

(c)

E

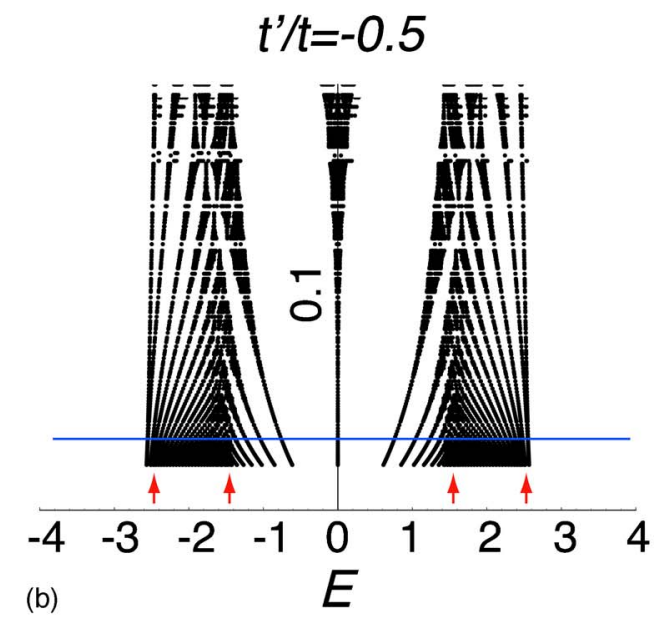

$t^{\prime} / t=+0.5$

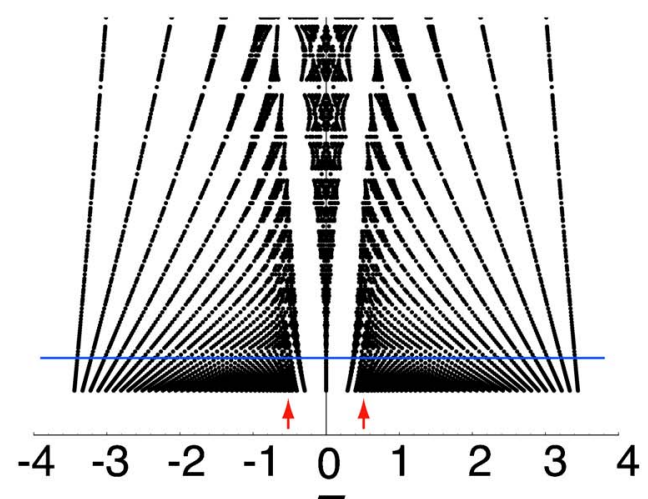

(d)

E

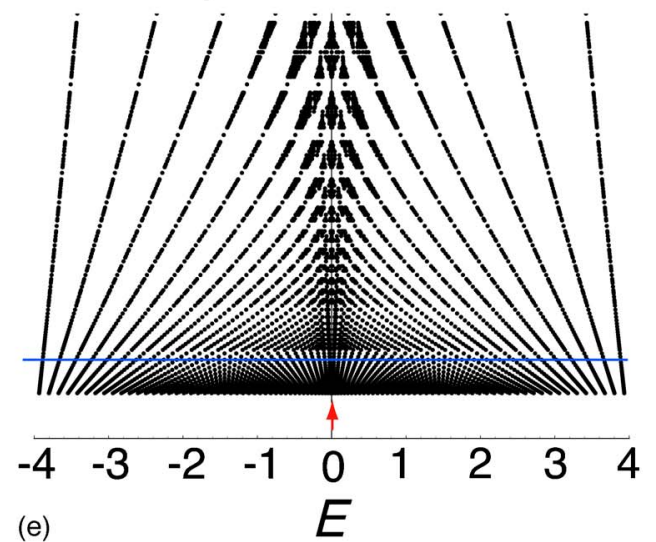

FIG. 5. (Color online) Blowup of Hofstadter's diagram in a weak magnetic field region for (a) $t^{\prime} / t=-1\left(\pi\right.$ flux), (b) $t^{\prime} / t=0.5$, (c) $t^{\prime} / t$ $=0$ (honeycomb), (d) $t^{\prime} / t=0.5$ and $t^{\prime} / t=1$ (square). The arrows indicate the positions of van Hove singularities. The blue lines indicate positions of a flux $\phi=1 / 31$, which corresponds to the one in Fig. 6.

$$
U_{\mu}\left(\boldsymbol{k}_{l}\right) \equiv\left|\operatorname{det} \boldsymbol{U}_{\mu}\left(\boldsymbol{k}_{l}\right)\right|^{-1} \operatorname{det} \boldsymbol{U}_{\mu}\left(\boldsymbol{k}_{l}\right)
$$

where

$$
\left(\boldsymbol{U}_{\mu}\right)_{i j}=\boldsymbol{\varphi}_{i}^{\dagger}\left(k_{l}\right) \boldsymbol{\varphi}_{j}\left(\boldsymbol{k}_{l}+\hat{\boldsymbol{\mu}}\right)(1 \leq i, j \leq n) .
$$

vortices" in the wave function, and we can always make the mesh avoid them with an infinitesimal shift. With the link variable we can define a lattice field strength by

$$
F_{12}\left(\boldsymbol{k}_{l}\right) \equiv \ln U_{1}\left(\boldsymbol{k}_{l}\right) U_{2}\left(\boldsymbol{k}_{l}+\hat{\mathbf{1}}\right) U_{1}\left(\boldsymbol{k}_{l}+\hat{\mathbf{2}}\right)^{-1} U_{2}\left(\boldsymbol{k}_{l}\right)^{-1},
$$

The link variables are well-defined except at singular points $\operatorname{det} \boldsymbol{U}_{\mu}\left(\boldsymbol{k}_{l}\right)=0$, which corresponding to "vortices" and "anti-

where the principal branch of the logarithm with $-\pi$ $<F_{12}\left(\boldsymbol{k}_{l}\right) / i \leq \pi$ is taken. The field strength is by definition 
invariant under gauge transformations, so that no specific gauge fixing is required. The Chern number on the lattice is defined as

$$
c_{F}\left(E_{F}\right)=\frac{1}{2 \pi i} \sum_{l} F_{12}\left(\boldsymbol{k}_{l}\right) .
$$

The Chern number thus computed is strictly an integer, since $\Sigma_{l} F_{12}$ just counts the number of vortices minus the number of antivortices in the Brillouin zone (i.e., the number of times the branches in ln are traversed), as has been proved in Ref. 47. Therefore, it should give the number in the continuum, $c_{F}$ appearing in Eq. (3) when the mesh in the Brillouin zone is sufficiently dense. This is due to the integral character of the lattice Chern number: For large $N_{\mu}$, we have

$$
U_{\mu}(\boldsymbol{k})=1+\operatorname{Tr} A_{\mu}\left(\boldsymbol{k}_{l}\right) \delta k_{\mu},
$$

with $A_{\mu}$ defined by Eq. (2), and we have

$$
\begin{aligned}
F_{12} & =\ln \prod_{\square}\left(1+\operatorname{Tr} A_{\mu} \delta k_{\mu}\right)+\mathcal{O}\left(|\delta k|^{3}\right) \\
& =\operatorname{Tr}\left[\partial_{1} A_{2}(k)-\partial_{2} A_{1}(k)\right] \delta k_{1} \delta k_{2}+\mathcal{O}\left(|\delta k|^{3}\right) .
\end{aligned}
$$

When coupled with Eq. (4), this reduces to Eq. (3), the Chern number in the continuum Brillouin zone.

\section{Dirac vs ordinary fermion quantization}

Let us now show the numerical results for the Chern number $c_{\mathrm{F}}$ in Eq. (4) as a function of the chemical potential $E_{F}$ for various values of $t^{\prime}$. While the integral Chern number $c_{\mathrm{F}}$ is defined only for each gap, we have plotted $c_{F}$ as step functions, which makes sense as long as the magnetic field is not too large, as in Fig. 6 with $\phi=1 / 31$, since the width of each Landau band is then much smaller than the size of gaps. We have displayed the values for spinless fermions.

The result shows a striking answer to one of our key questions: what is the fate of the Dirac-like behavior as we go away from $E=0$. When the Fermi energy is swept in the honeycomb lattice, the Dirac-like Hall conductivity steps of 2 (or 4 when spin degeneracy is included) in units of $-e^{2} / h$ exist around $E=0$ as has been noted by many authors. ${ }^{15-17,20,40}$ Let us call this Dirac-Landau behavior. Now, we can see that this Dirac-Landau behavior persists all the way up to some energy, which we identify to be the van Hove singularities appearing in Fig. 3. At these energies we have then a huge step accompanied by a sign change in the Hall conductivity. This result implies the following. The effective theory near the zero energy is Dirac-like fermions in the continuum limit, for which an unconventional quantization of the Hall conductivity has been derived from the Dirac Landau levels. ${ }^{15,16}$ The present calculation leads to the conclusion that the unusual property extends to an unexpectedly wide region of energy in the actual lattice fermion system. Outside the van Hove energies (i.e., in the band-edge regions), we recover the conventional QHE where the step changes by one in units of $-e^{2} / h$ (which we now call FermiLandau). This implies that a huge step accompanied by a sign change has to occur at the boundary between DiracLandau and Fermi-Landau behaviors, or the bands just at the van-Hove singularities induce a change, which is topological in that the relevant quantum numbers are topological.

A second striking feature in Fig. 6 is that the Dirac-like Hall conductivity $\propto(2 N+1)$ persists all along $-1 \leq t^{\prime} / t<1$ in the $\pi$-flux $\leftrightarrow$ honeycomb $\leftrightarrow$ square sequence, except at the square lattice. Namely, there always exists the Dirac-Landau behavior between the (innermost) van Hove singularities, except for the case when the singularities merge at $t^{\prime}=0$. We can check from the band dispersion (Fig. 2) that in this whole region we have two zero-mass Dirac cones in the Brillouin zone, which should cause this persistence of the anomalous QHE number.

We can now summarize the Hall conductivity for the spinless fermions on the honeycomb lattice for the entire energy region as

$$
\sigma_{x y}=-\frac{e^{2}}{h} \times\left\{\begin{array}{l}
+(N+1) E_{F}<-|t|, N=0,1,2, \ldots: \text { Landau level index counted from the band bottom, } \\
-(2 N+1)-|t|<E_{F}<0, N=0,1,2, \ldots \text { :Dirac-Landau level index counted from the zero energy, } \\
+(2 N+1) 0<E_{F}<+|t|, N=0,1,2, \ldots \text { :Dirac-Landau level index counted from the zero energy, } \\
-(N+1)|t|<E_{F}, N=0,1,2, \ldots \text { :Landau level index counted from the band top. }
\end{array}\right.
$$

We should again double these numbers if we include the spin degeneracy. While the unconventional quantization around the zero energy has been beautifully observed experimentally, ${ }^{19,20}$ pushing the chemical potential further to approach the van Hove energies should detect the topological phase transition.

We can note that, to be precise, there are in fact two sets of van Hove singularities [i.e., four in total, see Fig. 6(b)] in the honeycomb $\rightarrow \pi$-flux region. There, we have the DiracLandau behavior in the region between the innermost singularities, Fermi-Landau behavior in the next region, and another behavior outside the outermost singularities with the doubled Chern number $\propto 2 N$. Although this may seem a trivial detail, we can in fact make an interesting observation. Namely, we can raise a question: how the band-edge region can have doubled Chern number than Fermi-Landau 

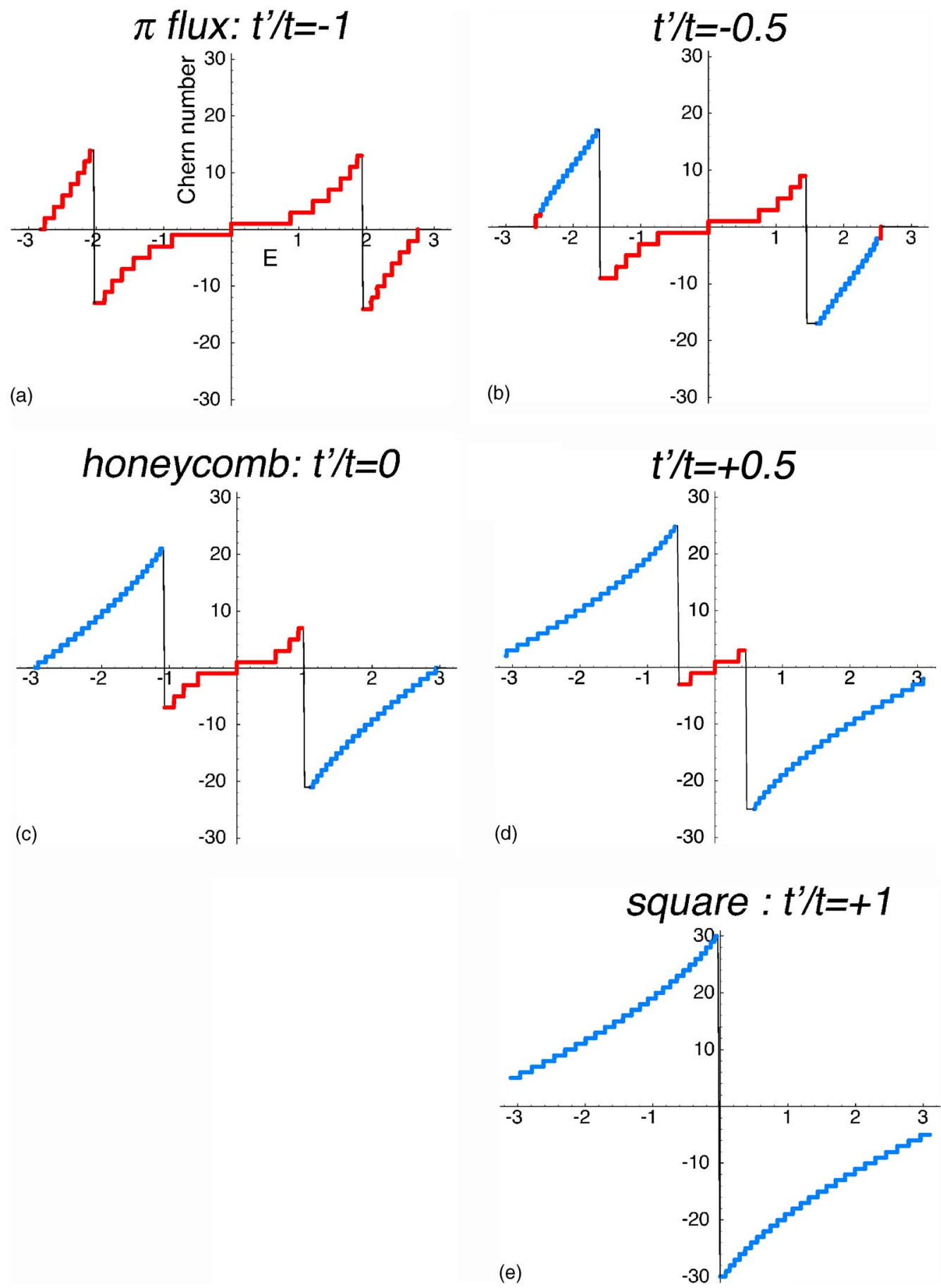

FIG. 6. (Color online) The Chern number (Hall conductivity in units of $-e^{2} / h$ ) for magnetic field $\phi=1 / 31$ is plotted against the Fermi energy $E_{F}$ : for (a) $t^{\prime} / t=-1$ ( $\pi$ flux), (b) $t^{\prime} / t=0.5$, (c) $t^{\prime} / t=0$ (honeycomb), (d) $t^{\prime} / t=0.5$, and (e) $t^{\prime} / t=1$ (square). The red lines indicate the steps of two in the Chern number sequence $\left[\sigma_{x y}= \pm(2 N+1) e^{2} / h, N\right.$ : integer], while the blue lines step of one $\left(\sigma_{x y}= \pm N e^{2} / h\right)$.

behavior? We can identify the origin of the doubled Chern numbers in the fermion band as the presence of two minima (maxima) in the dispersion in the Brillouin zone at the band bottom (top). So we can make an observation that a degenerate minima (or maxima) in the band dispersion can give rise to a Fermion species multiplication. We shall reinforce this view in terms of the topological equivalences in Sec. II B and edge state pictures in Sec. V.

\section{Degeneracy of Landau levels and the lattice effect}

Let us now look more closely at the distinction between the Dirac-Landau and Fermi-Landau levels in terms of the degeneracy of the Landau bands. The physical reason why we have a step of two in the quantized Hall conductivity for the Dirac-Landau levels can be ascribed, as in many other literatures, to the degeneracy (fermion doubling) of the 
bands. Let us take an example of $\phi=1 / 31$ employed in Fig. 6(c), for which we have $2 q=62$ eigenvalues $\epsilon_{j}(k)$. Among them, there are 7 pairs of twofold degenerate levels around $E=0$ with a total Chern number of 2 , while the other 48 levels are nondegenerate. Two of the nondegenerate Landau levels are located at the van Hove energies and carry a huge Chern number of -30 . It is such large numbers that require the accurate estimate with the present, lattice-gauge numerical algorithm.

The level adjacent to these have the ordinary Chern number of 1 each. So this abrupt change in the topological number defines the clear boundary. To be precise, however, we can note that approximate degeneracy in each two-fold Dirac-Landau levels (which is exact for a Dirac fermion with a rigorously linear dispersion) become slightly lifted toward the van Hove energy, while the gap across the next two-fold level becomes smaller, where the splitting $(0.05 t$ for $\phi$ $=1 / 31)$ and the gap $(0.02 t)$ become comparable (which is still much smaller than the thickness of the line that plots the energy dependence of the Chern number in Fig. 6, so the figure still makes sense). The deviation becomes more pronounced for stronger magnetic fields. We shall discuss this feature in Sec. V [see, e.g., Fig. 10(a) for $\phi=1 / 5$ ], where the gap can become comparable with the Landau band width.

\section{DIOPHANTINE EQUATION}

Having numerically calculated the Chern numbers, we can now raise the question: can we calculate these numbers algebraically? For square lattice systems, the Diophantine equation according to Thouless et $a l^{44}$ is known to be a simple yet powerful tool to compute the quantum Hall number of the Fermi seas. For the honeycomb lattice, however, the Diophantine equation has not been obtained. This is exactly where we can exploit the topological equivalence established in the previous sections to calculate the Chern numbers by the use of the Diophantine equation. Natural interest here is how the Dirac-Landau quantum Hall number of $(2 N+1)$ comes about. So we start with the Diophantine equation for the square lattice, where the Chern number $c_{J}$ of the Fermi sea composed of $J$ bands with flux per plaquette $\Phi=P / Q$ is given by the formula

$$
J \equiv P c_{J}(\bmod Q),\left|c_{J}\right| \leq Q / 2 .
$$

Now let us make its adiabatic continuation to the honeycomb lattice with magnetic field $\phi=1 / q$. Since the $\pi$-flux lattice is constructed here from the honeycomb lattice by the introduction of $t^{\prime} / t=-1$, the flux per square plaquette corresponds to $\phi / 2$ for the hexagonal plaquette with the shift in the period discussed in Sec. II B. Therefore, total flux per square plaquette of the $\pi$-flux lattice $\Phi$ reads

$$
\Phi=\frac{P}{Q}=\frac{1}{2}+\frac{\phi}{2}=\frac{q+1}{2 q},
$$

where the $1 / 2$ flux in the above equation is due to the $\pi$ flux. Let us first take the case of $q+1$ : prime ( $q$ : even). Then we have

$$
P=q+1, Q=2 q .
$$

Equation (5) together with Eq. (6) is a Diophantine equation for the honeycomb lattice with even $q$ valid in the Dirac fermion regime. To identify $J$ we assume for the honeycomb lattice that there exist a zero energy Dirac-Landau level, and the Dirac-Landau levels between the van Hove singularities are twofold degenerate.

Now let us calculate the Hall conductivity around the zero energy for the honeycomb lattice when $E_{F}$ lies in the $(N$ +1 )-th gap from the center (e.g., $N=0$ is the first gap above the zero energy). Note that the total number of the bands is $2 q$, so that the number of the negative-energy bands is $q-1$. Hence the number of bands below the $(N+1)$-th gap is $q$ $-1+2(N+1)$, i.e., $J=q+2 N+1$. From Eq. (5), the Diophantine equation then reads

$$
q+2 N+1 \equiv(q+1) c_{F}(\bmod 2 q),
$$

or

$$
(q+1)\left(c_{F}-1\right) \equiv 2 N \equiv 2 N(q+1)(\bmod 2 q) .
$$

This has an obvious solution

$$
c_{F}=2 N+1: E_{F}>0,
$$

and its particle-hole transformed $c_{F}=-(2 N+1)$ for $E_{F}<0$. Remarkably, this precisely gives the Chern number of the Dirac fermion, as computed in the previous section.

For an odd $q$, we put

$$
q=2 n+1, n=1,2, \ldots .
$$

Then the mapping to the $\pi$-flux lattice reads

$$
\Phi=\frac{P}{Q}=\frac{q+1}{2 q}=\frac{n+1}{2 n+1} .
$$

Namely, we have

$$
P=n+1, \quad Q=2 n+1 .
$$

Note that the total number of the bands in this case is $Q$ $=q=2 n+1$ rather than $2 q$ in the adiabatically transformed $\pi$ flux system. Then, the number of bands in the negative energy is $n$, and the number of bands below the $(N+1)$-th gap is $n+(N+1)$, i.e.,

$$
J=n+1+N,
$$

which gives, via Eqs. (5), (7), a Diophantine equation

$$
n+1+N \equiv(n+1) c_{F}(\bmod 2 n+1)
$$

or $(n+1)\left(c_{F}-1\right) \equiv N$. This expression is equivalent to $-n\left(c_{F}-1\right) \equiv-2 n N$. This has a solution $c_{\mathrm{F}}=2 N+1$, which gives the same Chern number for the prime $q+1$ case.

\section{EDGE VS BULK FOR THE HALL CONDUCTIVITY IN THE HONEYCOMB LATTICE}

When a system has a nontrivial topological structure, they should generically exhibit characteristic properties related to edge states. For the honeycomb lattice, edge states and their 
flat dispersion have been intensively discussed with its relevance to spin alignment. ${ }^{22,23}$ Another way of saying is that the honeycomb system has a bipartite symmetry, which guarantees the presence of the zero mode edge states, ${ }^{36}$ i.e., there exist macroscopic edge states at the zero energy unless the bipartite symmetry is broken. This gives rise to dispersionless edge states. The flat bands are unstable against bipartite symmetry breaking (Peierls instability), which is, e.g., realized when an antiferromagnetic spin ordering occurs. This may be viewed as a topological origin of the boundary spin moments. ${ }^{39}$

Now, the problem of edge states vs bulk states is particularly interesting for the QHE, since the problem of how the Dirac-Landau QHE $\propto(2 N+1)$ is related to the edge states is fundamentally interesting. One of the present authors has shown, for 2D square (or anisotropic square) systems, that the edge states whose energy dispersions run across the Landau bands have topological QHE numbers, for which

$$
\sigma_{x y}^{\text {edge }}=\sigma_{x y}^{\text {bulk }}
$$

by identifying the connection between the topological integers for the bulk and for the edge states. ${ }^{33,55}$ So let us now focus on the edge states of the honeycomb lattice in terms of the topological structure. It also allows us to clarify the bulkedge correspondence.

\section{A. Transfer matrix formalism}

\section{Transfer matrix}

We follow the analysis in Refs. 32, 33, and 55 to reduce the system to a one-dimensional model by making a partial Fourier transform of the fermion operators in the second direction,

$$
c_{\circ},(\mathbf{j})=\frac{1}{\sqrt{L_{2}}} \sum_{k_{2}} e^{i k_{2} j_{2}} c_{\circ}, \bullet\left(j_{1}, k_{2}\right) .
$$

This yields a $k_{2}$-dependent series of one-dimensional Hamiltonian

$$
\begin{gathered}
H=\sum_{k_{2}} H_{1 \mathrm{D}}\left(k_{2}\right), \\
H_{1 \mathrm{D}}\left(k_{2}\right)=\sum_{j_{1}}\left[t_{\bullet \bullet}\left(j_{1}, k_{2}\right) c_{\diamond}^{\dagger}\left(j_{1}, k_{2}\right) c_{\bullet}\left(j_{1}, k_{2}\right)\right. \\
+t_{\bullet}\left(j_{1}, k_{2}\right) c_{\bullet}^{\dagger}\left(j_{1}+1, k_{2}\right) c_{\bullet}\left(j_{1}, k_{2}\right)+\text { H.c. }
\end{gathered}
$$

where $k_{2}$-dependent hopping parameters are

$$
\begin{gathered}
t_{\circ}\left(j_{1}, k_{2}\right)=t\left(1+e^{i k_{2}-i 2 \pi \phi j_{1}}\right), \\
t_{\bullet \circ}\left(j_{1}, k_{2}\right)=t\left[1+\left(t^{\prime} / t\right) e^{i k_{2}-i 2 \pi \phi\left(j_{1}+1 / 2\right)}\right] .
\end{gathered}
$$

Expanding one-particle eigenstates $\left|E, k_{2}\right\rangle$ with energy $E$ and momentum $k_{2}$ as

$$
\begin{aligned}
\left|E, k_{2}\right\rangle= & \sum_{j_{1}}\left[\psi_{\bullet}\left(E, j_{1}, k_{2}\right) c_{\bullet}^{\dagger}\left(j_{1}, k_{2}\right)|0\rangle\right. \\
& \left.+\psi_{\circ}\left(E, j_{1}, k_{2}\right) c_{\circ}^{\dagger}\left(j_{1}, k_{2}\right)|0\rangle\right],
\end{aligned}
$$

we find that the Schrödinger equation $H_{1 \mathrm{D}}\left(k_{2}\right)\left|E, k_{2}\right\rangle$ $=E\left|E, k_{2}\right\rangle$ is cast into a matrix form

$$
\begin{aligned}
& \left(\begin{array}{l}
\psi_{0}\left(j_{1}\right) \\
\psi_{\bullet}\left(j_{1}\right)
\end{array}\right)=M_{\bullet \bullet}\left(j_{1}\right)\left(\begin{array}{l}
\psi_{\bullet}\left(j_{1}\right) \\
\psi_{0}\left(j_{1}-1\right)
\end{array}\right), \\
& \left(\begin{array}{l}
\psi_{\bullet}\left(j_{1}+1\right) \\
\psi_{\circ}\left(j_{1}\right)
\end{array}\right)=M_{\bullet \circ}\left(j_{1}\right)\left(\begin{array}{l}
\psi_{0}\left(j_{1}\right) \\
\psi_{\bullet}\left(j_{1}\right)
\end{array}\right),
\end{aligned}
$$

with

$$
\begin{aligned}
M_{\bullet \bullet}\left(j_{1}\right) & =\left(\begin{array}{ll}
\frac{E}{t_{\bullet \bullet}^{*}\left(j_{1}\right)} & -\frac{t_{\bullet \circ}\left(j_{1}-1\right)}{t_{\circ \bullet}^{*}\left(j_{1}\right)} \\
1 & 0
\end{array}\right) M_{\bullet \circ}\left(j_{1}\right) \\
& =\left(\begin{array}{ll}
\frac{E}{t_{\bullet \circ}^{*}\left(j_{1}\right)} & -\frac{t_{\circ \bullet}\left(j_{1}\right)}{t_{\bullet \circ}^{* *}\left(j_{1}\right)} \\
1 & 0
\end{array}\right) .
\end{aligned}
$$

Therefore, we have

$$
\left(\begin{array}{l}
\psi_{\bullet}\left(j_{1}+1\right) \\
\psi_{0}\left(j_{1}\right)
\end{array}\right)=M_{\mathrm{t}}\left(j_{1}\right)\left(\begin{array}{l}
\psi_{\bullet}\left(j_{1}\right) \\
\psi_{0}\left(j_{1}-1\right)
\end{array}\right)
$$

with

$$
M_{\mathrm{t}}\left(j_{1}\right)=M_{\bullet}\left(j_{1}\right) M_{\bullet \bullet}\left(j_{1}\right),
$$

where every quantity is a function of $\left(E, j_{1}, k_{2}\right)$. They are a set of equations for $2 L_{1}-1$ variables $\psi_{0}\left(j_{x}\right)\left(j_{x}=1, \ldots, L_{x}\right.$ $-1), \psi_{0}\left(j_{x}\right)\left(j_{x}=1, \ldots, L_{x}\right)$ with open boundary conditions $\psi_{0}(0)=\psi_{0}\left(L_{1}\right)=0$ (see Fig. 7). To impose this condition, we consider cylindrical systems with a zigzag edge at one end and a bearded (or Klein's) edge at the other as illustrated in Fig. 7. This is just a technical convention to apply the following transfer-matrix formalism. In terms of the energy spectrum, zigzag-bearded system has an $E=0$ edge state in zero magnetic field that extend over the entire Brillouin zone, as was first pointed out in Ref. 56. In passing, we mention that the local transformation

$$
\begin{gathered}
\psi_{\bullet}\left(E, j_{1}\right) \rightarrow-\psi_{\bullet}\left(E, j_{1}\right)=\psi_{\bullet}\left(-E, j_{1}\right), \\
\psi_{0}\left(E, j_{1}\right) \rightarrow \psi_{0}\left(E, j_{1}\right)=\psi_{0}\left(-E, j_{1}\right),
\end{gathered}
$$

yields the same Schrodinger equation but with $-E$. This is due to the bipartite symmetry of honeycomb: Eigenstates for $\pm E$ are paired with the same amplitudes $\left|\psi_{\bullet, 0}(m)\right|$ except for the zero energy.

Here note the periodicity $M_{s}\left(j_{1}+q\right)=M_{s}\left(j_{1}\right)$ for $s=0 \bullet, s$ $=\bullet$ o, and hence $M_{\mathrm{t}}\left(j_{1}+q\right)=M_{\mathrm{t}}\left(j_{1}\right)$. We can now define the transfer matrix for a unit period

$$
M\left(E, k_{2}\right)=\prod_{j_{1}=1}^{q} M_{\mathrm{t}}\left(E, j_{1}, k_{2}\right),
$$



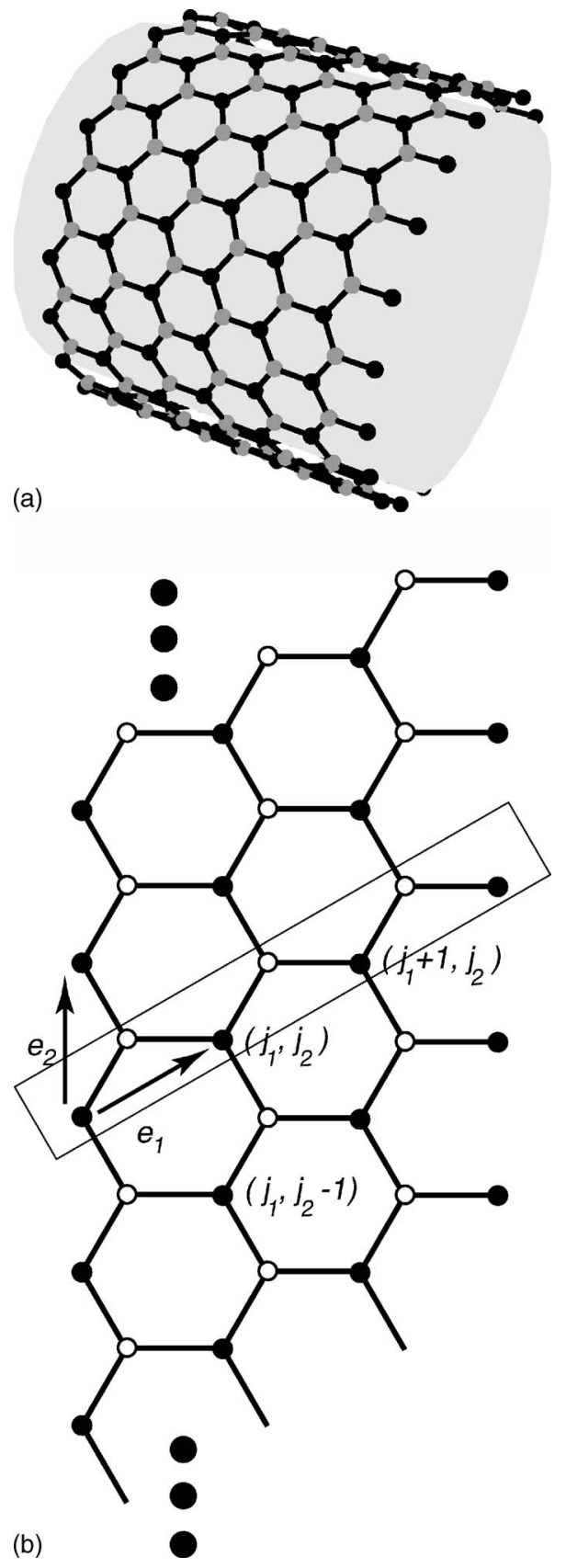

FIG. 7. A cylindrical system with zigzag and bearded edges.

then

$$
\left(\begin{array}{c}
\psi_{\bullet}(q+1) \\
\psi_{\circ}(q)
\end{array}\right)=M\left(\begin{array}{l}
\psi_{\bullet}(1) \\
\psi_{\circ}(0)
\end{array}\right) .
$$

For $L_{1}=q l$ with an integer $l$ we have

$$
\left(\begin{array}{l}
\psi_{\bullet}\left(L_{1}+1\right) \\
\psi_{\circ}\left(L_{1}\right)
\end{array}\right)=M^{l}\left(\begin{array}{l}
\psi_{\bullet}(1) \\
\psi_{\circ}(0)
\end{array}\right),
$$

with the boundary condition $\psi_{\circ}(0)=\psi_{\circ}\left(L_{1}\right)=0$ and $\psi_{\bullet}(1)=1$, which is equivalent to an algebraic equation $\left(M^{l}\right)_{21}(E)=0$ for $E$, which is a polynomial of order $2 L_{1}-1$ and has as many real roots.

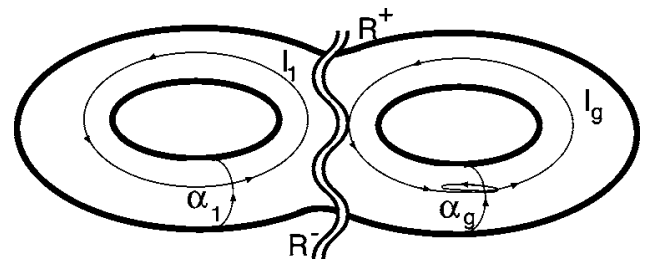

FIG. 8. A Riemann surface $\Sigma_{2 q-1}\left(k_{2}\right)$ for the Bloch function which is a complex energy surface for defining $\rho(E)$ consistently. Its genus is $2 q-1$, which coincides with the number of energy gaps.

We can extract the edge states from the whole spectrum. ${ }^{32,33}$ Namely, $2 q-1$ roots of $M_{21}(E)=0$, a polynomial equation of order $2 q-1$, give the edge state energies, $E_{j}\left(k_{2}\right)$ for $j=1, \ldots, 2 q-1$. By contrast, usual cylindrical systems where both edges are zigzag, bearded, or armchair do not allow such extraction. For example, in the case for zigzag edges, the boundary condition is replaced by $\psi_{\circ}(0)$ $=\psi \cdot\left(L_{1}+1\right)=0$ in the present formulation. Hence, the total spectra is determined by $\left(M^{l}\right)_{11}(E)=0$, giving $2 L_{1}$ real roots. Edge states are contained in these spectra, but there is no simple way to directly extract them only.

A remarkable feature here is that, on top of the edge states across adjacent Landau bands, there always exist exactly constant $E=0$ edge states, since the number of the edge states are odd 2q-1. Namely, a system with zigzag edges has a zero-energy edge mode in some region of $k_{2}$, a system with bearded edges in the other region, and the present system has the mode over the entire region. This is a simple extension of the discussion in the absence of a magnetic field. ${ }^{36}$

We can further identify the spatial position of edge states: With $M_{11}\left(E_{j}\right)$, we can show that

$$
\begin{aligned}
& \left|M_{11}\left(E_{j}\right)\right|<1 \text { :localized at the zigzag edge, } \\
& \left|M_{11}\left(E_{j}\right)\right|>1 \text { :localized at the bearded edge. }
\end{aligned}
$$

With Laughlin's argument, ${ }^{30}$ supplemented by the above behavior of the edge state (as a function of the momentum $k_{2}$ ), one can identify a topological number $I\left(E_{F}\right)$, the number of electrons carried by Laughlin's adiabatic procedure, for each edge state branch when the chemical potential $E_{F}$ traverses the branch. ${ }^{30,32,33,55}$

Having determined the spectra for the edge states with the transfer matrix, we can now relate the edge states to the Bloch functions of the bulk systems: Let us relax the open boundary condition, and impose instead a periodic boundary condition $H\left(j_{1}+q, k_{2}\right)=H\left(j_{1}, k_{2}\right)$ on the local Hamiltonian (9). Then the Bloch theorem for the bulk state leads to $\psi \cdot\left(j_{1}+q\right)=e^{i k_{1} q} \psi_{\bullet}\left(j_{1}\right)$ and $\psi_{0}\left(j_{1}+q\right)=e^{i k_{1} q} \psi_{0}\left(j_{1}\right)$ or

$$
\left(\begin{array}{l}
\psi_{\bullet}\left(j_{1}+q+1\right) \\
\psi_{0}\left(j_{1}+q\right)
\end{array}\right)=e^{i k_{1} q}\left(\begin{array}{l}
\psi_{\bullet}\left(j_{1}+1\right) \\
\psi_{0}\left(j_{1}\right)
\end{array}\right) .
$$

Comparing this with Eq. (14), we see that the eigenvalue of the transfer matrix $M$ is $e^{i k_{1} q}$, i.e., just a phase factor. Therefore, the energy bands are specified by the following condition for the eigenvalue $\rho$ of the transfer matrix $M, \operatorname{det}(\rho 1$ $-M)=0$, with $|\rho|=1$. As shown in the Appendix B, the condition for the energy band is given by $|\operatorname{Tr} M| \leq 2$. 

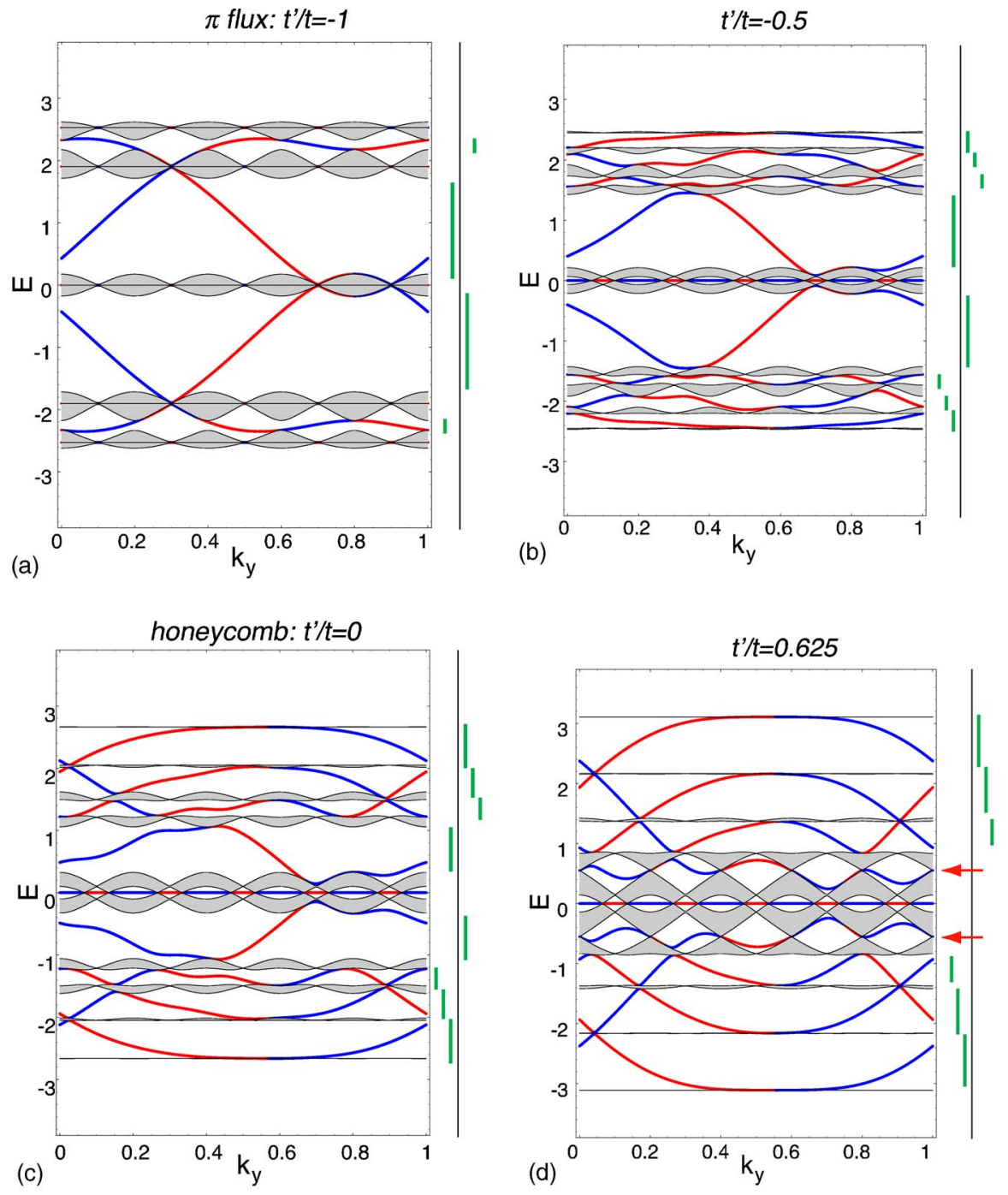

FIG. 9. (Color online) The energy spectra for the cylindrical system in Fig. 7 with the flux $\phi$ $=1 / 5$ for (a) $t^{\prime} / t=-1$ ( $\pi$-flux), (b) $t^{\prime} / t=0.625$, (c) $t^{\prime} / t=0$ (honeycomb), (d) $t^{\prime} / t=0.5$, and (e) $t^{\prime} / t$ $=1$ (square). Blue (red) lines are edge states localized at the bearded (zigzag) edges, while the bulk energy bands are shown as grey regions. The topological number $I\left(E_{F}\right)$, when $E_{F}$ is in a major gap in the negative energies are (a) $2,-1$; (b) $1,2,3,-1$; (c) 1 , 2, 3, -1; (d) 1, 2, 3, (ill-defined); and (e) 1, 2, 3, 4. A topological transition accompanied with a discrete Chern number change by $\Delta c=-5$ (from -4 to +1 ) at the fourth major gap occurs between $t^{\prime} / t=0.5 \rightarrow 1$. The green lines in the right are the topological numbers $I\left(E_{F}\right)$ obtained by counting the edge states.

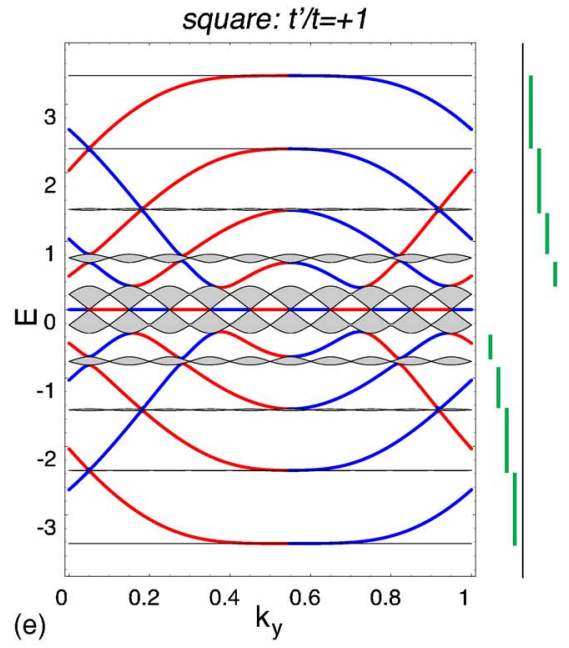

The eigenvalue of the transfer matrix is also written as $\rho=\frac{1}{2}\left\{\operatorname{Tr} M-\left[(\operatorname{Tr} M)^{2}-4 \operatorname{det} M\right]^{1 / 2}\right\}$, where we have to fix the branch of the square root. Then the Riemann surface $\Sigma_{2 q-1}$ of the Bloch function is given by that of the eigenvalue $\rho(E)$ and its genus is generically $2 q-1$ which are the number of energy gaps (Fig. 8). With this Riemann surface and the ar- guments in Refs. 32, 33, and 55, one can establish the bulkedge correspondence as

$$
c_{F}\left(E_{F}\right)=I\left(E_{F}\right),
$$

where $c_{F}\left(E_{F}\right)$ is the Chern number obtained in Sec. III, while $I\left(E_{F}\right)$ is the topological number for edge states which corre- 

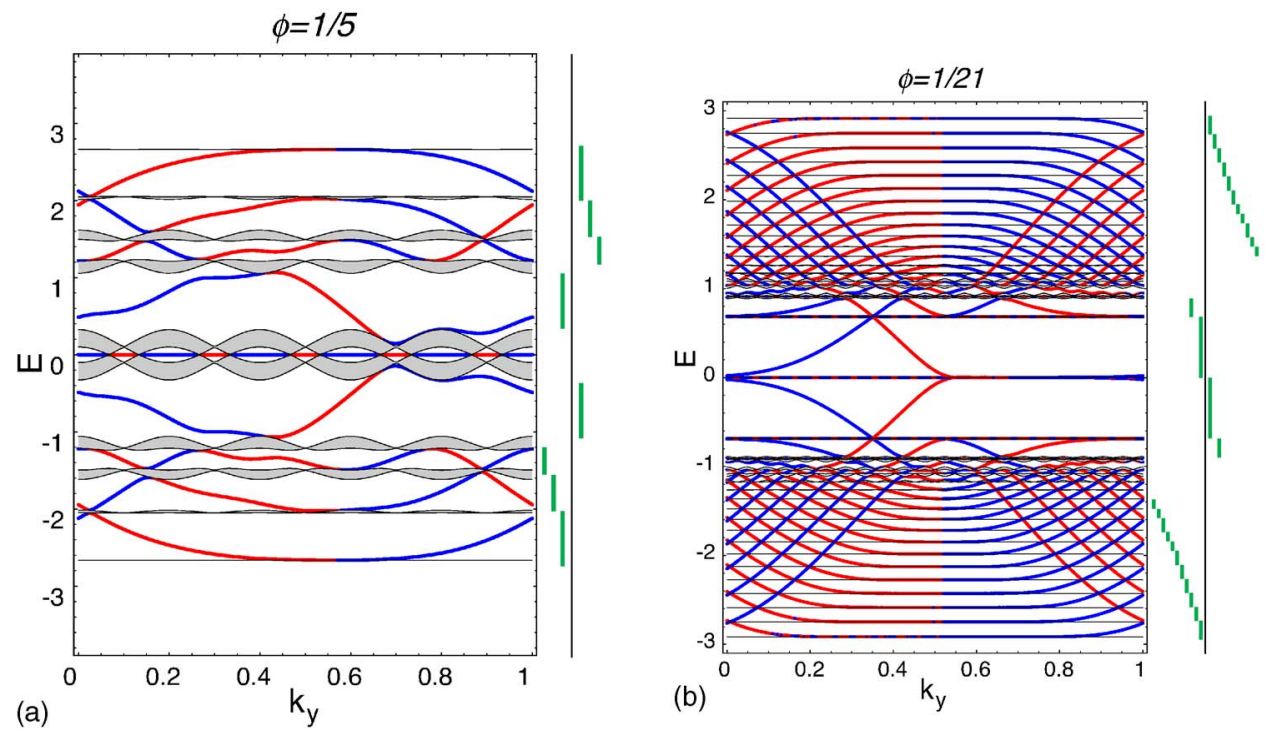

FIG. 10. (Color online) The energy spectra of the honeycomb lattice with zigzag and bearded edges for (a) $\phi=1 / 5$ and (b) $\phi$ $=1 / 21$. (c) is a blowup of (b) and (d) is for $\phi=1 / 51$. The topological number $I\left(E_{F}\right)$ when $E_{F}$ is in a major energy gaps is also indi-
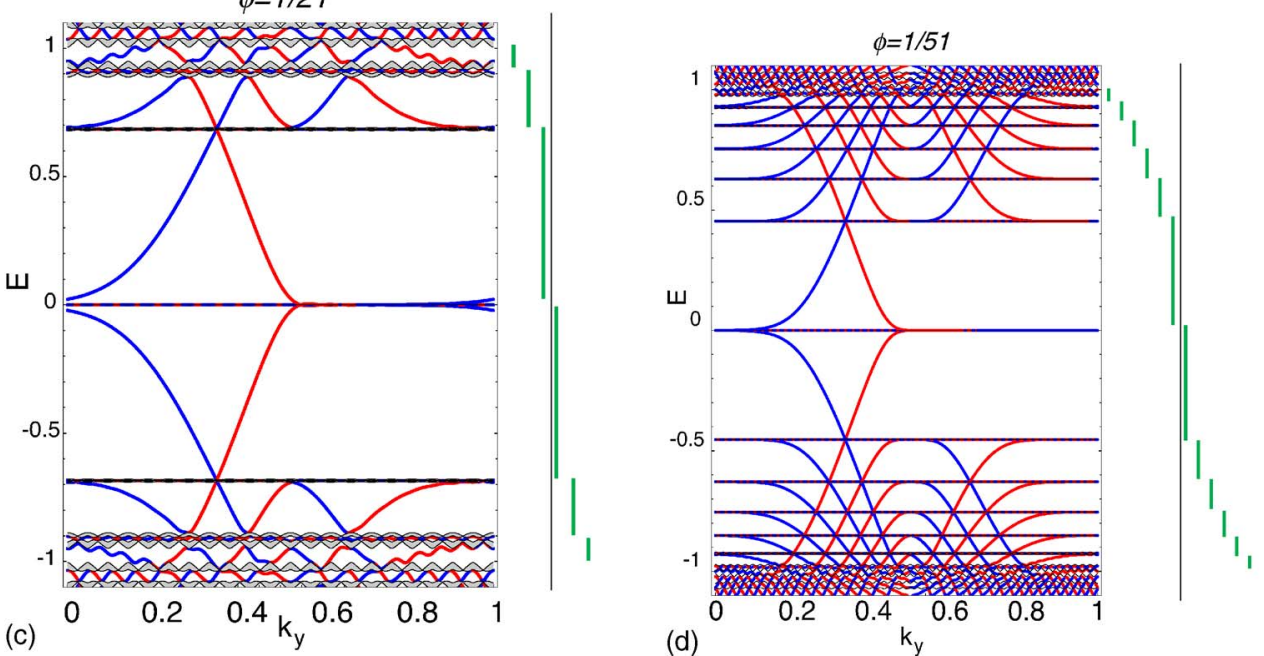
cated next to the vertical axis.

sponds to the number of intersections on the Riemann surface $\Sigma_{2 q-1}$.

\section{B. Edge-bulk equivalence and topological transition}

Before we discuss the experimental situation, let us look at the case of strong magnetic fields with $\phi=1 / q$ with $q$ $=O(1)$. This case, while unrealistic, is useful for heuristic purposes. In Fig. 9, the spectra of the cylindrical systems are shown for a relatively large $\phi=1 / 5$. The shaded regions are Landau bands, while the blue (red) curves across the bands correspond to edge states localized at the bearded (zigzag) edges. It should be noted that we always find a dispersionless edge state at the zero energy, which is due to bipartite symmetry. This is the state employed for the boundary magnetic moment, but does not contribute to the topological number, since the number is ill defined due to the gap closing at the zero energy.

For the honeycomb lattice there are ten bands ( with two center bands touching with each other) for $\phi=1 / 5$, as observed in Fig. 9(c). By counting the number of the edge states, we can see that the topological numbers $I\left(E_{F}\right)$ are 1,2 ,
$3,-1,+1,-3,-2$, and -1 , when $E_{F}$ lies in the $j$ th major gap for $j=1, \ldots, 8$, respectively. Note that gap-closing occurs at the zero energy, and the increment of the topological number across these degenerate bands is $\Delta I=2$ which corresponds to the Dirac-Landau level quantization of the Hall conductivity discussed in Sec. III.

If we move on to $\pi$-flux lattice $\left(t^{\prime} / t: 0 \rightarrow-1\right)$, the Dirac fermion level with $\Delta I=2$ at the zero energy persists [Figs. 9 (a) and 9(b)]. This is the edge-bulk correspondence version of the topological equivalence (I) in Sec. II B. If we move on to square lattice $\left(t^{\prime} / t: 0 \rightarrow 1\right)$, on the other hand, we can observe a topological change in these bands. At $t^{\prime} / t=1$, the topological numbers $I\left(E_{F}\right)$ reads $1,2,3,4,-4,-3,-2,-1$ versus $E_{F}$, which dictates that $E=0$ is now a van Hove energy with $|\Delta I|=8 \gg 1$. This corresponds to the fact that vanHove energies merge to wash out the Dirac-Landau region precisely at $t^{\prime} / t=1$, so the square lattice is singular in this respect. The merging of the relevant Landau bands (fourthsixth for $\phi=1 / 5)$ is clearly seen in the spectra at $t^{\prime} / t$ $=0.625$ shown in Fig. 9(d), where the topological number -5 moves to the zero energy level from the levels just below and above it. 
We next investigate the second equivalence 2 given in Sec. III via similar observations. Let us start with the honeycomb lattice. The increment in the topological number is $\Delta I=1$ in the band edge regions, the conventional FermiLandau quantization. This feature indeed survives in the adiabatic process $t^{\prime} / t: 0 \rightarrow 1$ in this region. This is the topological equivalence 2 . On the other hand, for $t^{\prime} / t: 0 \rightarrow-1$ these bands merge in pairs to produce doubled Chern numbers, which corresponds to the fermion doubling due to the multiple band extrema discussed in Sec. III C. In passing, we mention that one can confirm the particle-hole symmetry, i.e., the bipartite symmetry in the topological numbers

$$
I\left(E_{F}\right)=-I\left(-E_{F}\right) \text {. }
$$

\section{From strong to weak magnetic fields}

Having identified the strong-field behavior, let us next show how the spectra changes when the magnetic field has smaller, more realistic values. In Fig. 10, we show the spectra for the honeycomb lattice. The weaker the external magnetic field becomes, the narrower the gaps as well as the band widths become: For $\phi=1 / 31$, the bands look similar to Landau levels, but the edge states continue to exhibit characteristic behavior around the zero energy region $(1,3,5, \ldots$, edge branches for the consecutive gaps), and this region is separated by the van Hove energies $\sim \pm 1$, outside of which we recover the free fermionic topological numbers with $\Delta I$ $=1$. As discussed above, in strong magnetic fields such as $\phi=1 / 5$, only two bands across the zero energy are DiracLandau levels, while the next bands with energy $\sim \pm 1$ are van Hove bands. Decreasing magnetic field makes the number of Dirac fermion bands increases: The number of edge states in Fig. 10 is precisely in accord with this.

\section{SUMMARY AND DISCUSSIONS}

In summary, we have shown that the Landau levels are divided by the van Hove singularities into two regimes. One is effectively described by Dirac particles, and the other by ordinary finite-mass fermions. Remarkably, this persists as we convert the lattice into square or $\pi$-flux lattices. We have confirmed this both from the Chern number argument on Hofstadter's diagram and by the bulk-edge correspondence.

The van Hove singularities have turn out to play a key role in separating the Dirac-Landau and Fermi-Landau regions. Thus the anomalous QHE is not unique to the honeycomb lattice, but continuously shared by a class of lattices (except by the square lattice). The Dirac-Landau behavior can also occur when there are multiple extrema in the dispersion, as in the region between honeycomb and $\pi$-flux lattices.

The properties revealed here for the whole spectrum of the honeycomb lattice may be experimentally observable if the chemical potential can be varied over a wide range. In real graphene samples there may be disorder, in which case we are talking about a dirty Hofstadter system. However, non-monotonic behaviors should survive the disorder as far as the degree of disorder is not too large, as has been indi- cated by a numerical calculation for a dirty Hofstadter system. ${ }^{57}$ As for the $\pi$-flux lattice, the model effectively describes the excitation spectrum of $d$-wave superconductors, so there may exist a relevance.

Note added. After this work was submitted, a preprint appeared with some overlap with ours. ${ }^{58}$

\section{ACKNOWLEDGMENTS}

This work was in part supported by Grants in Aids from the Japanese Ministry of Education (for Creative Scientific Research Project "Quantum properties of organic-inorganic complex structures" for HA, Grant-in-Aid for Scientific Research (Grant No. 17540347) from JSPS and on Priority Areas (Grant No. 18043007) from MEXT for YH and Grantin-Aid for Scientific Research (Grant No. 18540365) from JSPS for T.F. Y.H. was also supported in part by the Sumitomo foundation.

\section{APPENDIX A: MOMENTUM REPRESENTATION OF THE HAMILTONIAN}

We derive the Hamiltonian for the honeycomb lattice in the momentum representation for the flux per hexagon $\phi$ $=p / q$, where $p$ and $q$ are mutually prime integers. We label the unit cell as $\boldsymbol{j}=j_{1} \boldsymbol{e}_{1}+j_{2} \boldsymbol{e}_{2}$. Let $\boldsymbol{r}_{\mu}$ be the reciprocal vectors (divided by $2 \pi$ ) satisfying $\boldsymbol{e}_{\mu} \cdot \boldsymbol{r}_{\nu}=\delta_{\mu \nu}$. In an external magnetic field, it is convenient to introduce a larger unit cell along coordinate 1 as $J=J_{1}\left(q e_{1}\right)+J_{2} e_{2}$. In this unit cell, the Fermi operators are denoted as $c_{s m}(J)$ with $s=\bullet, \circ$ and $m$ $=1, \ldots, q$. Let $\boldsymbol{K}$ be the corresponding momentum defined by $\boldsymbol{K}=K_{1}\left(\boldsymbol{r}_{1} / q\right)+K_{2} \boldsymbol{r}_{2}$. Then the Fourier transformation can be defined as

$$
c_{s m}(\boldsymbol{J})=\int_{0}^{2 \pi} \frac{d^{2} K}{(2 \pi)^{2}} e^{i K \cdot \mathbf{J}} c_{s m}(\mathbf{K}) .
$$

Note that $K_{\mu} \in[0,2 \pi]$. Introduce $K_{1} / q=k_{1}$ and $K_{2}=k_{2}$, where $k_{\mu}$ denotes the momentum in zero magnetic field. It is convenient to introduce operators $\boldsymbol{c}^{\dagger}(k)$ $=\left[c_{\bullet_{1}}^{\dagger}(k), c_{\circ 1}^{\dagger}(k), c_{\bullet_{2}}^{\dagger}(k), c_{\circ 2}^{\dagger}(k), \ldots, c_{\bullet q}^{\dagger}(k), c_{\circ q}^{\dagger}(k)\right]$. With these we obtain the Hamiltonian in the momentum representation Eq. (1) as

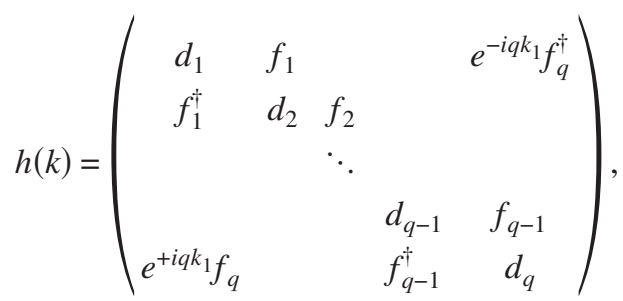

where

$$
\begin{aligned}
& d_{j}\left(k_{2}\right)=t\left(\begin{array}{ll}
0 & 1+e^{-i k_{2}+i 2 \pi \phi j} \\
1+e^{i k_{2}-i 2 \pi \phi j} & 0
\end{array}\right), \\
& f_{j}\left(k_{2}\right)=t\left(\begin{array}{ll}
0 & 0 \\
1+\left(t^{\prime} / t\right) e^{-i k_{2}+i 2 \pi \phi(j+1 / 2)} & 0
\end{array}\right) .
\end{aligned}
$$




\section{APPENDIX B: GAUGE TRANSFORMATION AND THE TRANSFER MATRIX}

In the transfer matrix formalism, the energy bands of the bulk system can be determined by the condition

$$
\rho^{2}-\rho \operatorname{Tr} M+\operatorname{det} M=0
$$

that satisfies $|\rho|=1$. Since the transfer matrix has complex matrix elements, let us separate the hopping parameters into the magnitude and the phase as

$$
t_{s}\left(j_{1}, k_{2}\right)=r_{s}\left(j_{1}, k_{2}\right) e^{i \phi_{s}\left(j_{1}, k_{2}\right)}, \quad s=\circ \bullet, \bullet,
$$

where $r_{s}$ is a real non-negative parameter. Obviously, $r_{s}\left(j_{1}\right.$ $\left.+q, k_{2}\right)=r_{s}\left(j_{1}, k_{2}\right)$. In what follows, the $k_{2}$ dependences are suppressed for simplicity.

Then the matrices in Eq. (10) can be expressed as

$$
\begin{aligned}
M_{\circ}\left(j_{1}\right)= & \left(\begin{array}{cc}
e^{i \phi_{\circ}\left(j_{1}\right)+i \phi_{\circ}\left(j_{1}-1\right)} & 0 \\
0 & e^{i \phi_{\circ}\left(j_{1}-1\right)}
\end{array}\right) \\
& \times\left(\begin{array}{cc}
\frac{E}{r_{\circ}\left(j_{1}\right)} & -\frac{r_{\circ}\left(j_{1}-1\right)}{r_{\circ}\left(j_{1}\right)} \\
1 & 0
\end{array}\right)\left(\begin{array}{cc}
e^{-i \phi_{\circ}\left(j_{1}-1\right)} & 0 \\
0 & 1
\end{array}\right),
\end{aligned}
$$

$$
\begin{aligned}
M_{\bullet \circ}\left(j_{1}\right)= & \left(\begin{array}{cc}
e^{i \phi_{\bullet \bullet}\left(j_{1}\right)+i \phi_{\bullet \circ}\left(j_{1}\right)} & 0 \\
0 & e^{i \phi_{\bullet \circ}\left(j_{1}\right)}
\end{array}\right)\left(\begin{array}{cc}
\frac{E}{r_{\bullet \circ}\left(j_{1}\right)} & -\frac{r_{\circ \bullet}\left(j_{1}\right)}{r_{\bullet \circ}\left(j_{1}\right)} \\
1 & 0
\end{array}\right) \\
& \times\left(\begin{array}{cc}
e^{-i \phi_{\bullet \circ}\left(j_{1}\right)} & 0 \\
0 & 1
\end{array}\right) .
\end{aligned}
$$

By multiplying the above two matrices, we can see that the transfer matrix (11) can be written as

$$
\begin{aligned}
M_{\mathrm{t}}\left(j_{1}\right)= & \left(\begin{array}{cc}
e^{i \phi_{\bullet \bullet}\left(j_{1}\right)+i \phi_{\bullet \circ}\left(j_{1}\right)} & 0 \\
0 & e^{i \phi_{\bullet \bullet}\left(j_{1}\right)}
\end{array}\right)\left(\begin{array}{cc}
\frac{E}{r_{\bullet \circ}\left(j_{1}\right)} & -\frac{r_{\circ \bullet}\left(j_{1}\right)}{r_{\bullet \circ}\left(j_{1}\right)} \\
1 & 0
\end{array}\right) \\
& \times\left(\begin{array}{cc}
\frac{E}{r_{\circ \bullet}\left(j_{1}\right)} & -\frac{r_{\circ}\left(j_{1}-1\right)}{r_{\circ \bullet}\left(j_{1}\right)} \\
1 & 0
\end{array}\right)\left(\begin{array}{cc}
1 & 0 \\
0 & e^{i \phi_{\bullet \circ}\left(j_{1}-1\right)}
\end{array}\right) \\
= & U\left(j_{1}+1\right) \tilde{M}_{\mathrm{t}}\left(j_{1}\right) U^{-1}\left(j_{1}\right),
\end{aligned}
$$

where $\tilde{M}_{\mathrm{t}}$ is a real matrix defined by the product of the second and third matrices in the right-hand side of the first line, and $U\left(j_{1}\right)$ is a diagonal matrix defined by

$$
U\left(j_{1}\right)=\exp i\left(\begin{array}{cc}
\sum_{j_{1}^{\prime}}^{j_{1}-1}\left[\phi_{\bullet \bullet}\left(j_{1}^{\prime}\right)+\phi_{\bullet \bullet}\left(j_{1}^{\prime}\right)\right] & 0 \\
0 & \sum_{j_{1}^{\prime}}^{j_{1}-1} \phi_{\bullet \bullet}\left(j_{1}^{\prime}\right)+\sum_{j_{1}^{\prime}}^{j_{1}-2} \phi_{\bullet \bullet}\left(j_{1}^{\prime}\right)
\end{array}\right) .
$$

Here, the sum over $j_{1}^{\prime}$ starts from some integer, say, -1 . The matrix $U\left(j_{1}\right)$ is nothing but a local gauge transformation for the wave functions $\phi_{0}\left(j_{1}\right)$ and $\psi_{0}\left(j_{1}\right)$. Therefore, we arrive at an expression for the unit cell transfer matrix (13),

$$
M=U(q+1) \tilde{M} U^{-1}(1),
$$

where $\tilde{M}$ is a real matrix defined by $\tilde{M} \equiv \prod_{j_{1}=1}^{q} \tilde{M}_{\mathrm{t}}\left(j_{1}\right)$. Note that

$$
U^{-1}(1) U(q+1)=e^{i \Theta} \mathbf{1},
$$

where $\Theta$ is defined by $\Theta \equiv \sum_{j_{1}=1}^{q} \Sigma_{s} \phi_{s}\left(j_{1}\right)$ $=\sum_{j_{1}=1}^{q} \operatorname{Arg} t_{\bullet}\left(j_{1}\right) t_{\circ \bullet}\left(j_{1}\right)(\bmod 2 \pi)$. Finally, we have

$$
\operatorname{det} M=e^{2 i \Theta},
$$

$$
\operatorname{Tr} M=e^{i \Theta} \operatorname{Tr} \tilde{M},
$$

where we have used $\operatorname{det} \tilde{M}=1$ in the first line. It should be stressed again that $\operatorname{Tr} \tilde{M}$ is real. Thus we conclude that the quadratic equation has solutions that satisfy $|\rho|=1$ if $|\operatorname{Tr} \tilde{M}|=|\operatorname{Tr} M| \leq 2$.

As a function of the energy, the eigenvalue of the transfer matrix is written as

$$
\rho=\frac{1}{2}\left\{\operatorname{Tr} M-\left[(\operatorname{Tr} M)^{2}-4 \operatorname{det} M\right]^{1 / 2}\right\},
$$

where we have to fix the branch of the square root. Then the Riemann surface $\Sigma_{2 q-1}$ of the Bloch function is given by that of the eigenvalue $\rho(E)$ and its genus is generically $2 q-1$ which are the number of energy gaps. 
${ }^{1}$ T. Eguchi, P. B. Gilkey, and A. J. Hanson, Phys. Rep. 66, 213 (1980).

${ }^{2}$ Current Algebra and Anomaly, edited by S. B. Treiman, R. Jackiw, B. Zumino, and E. Witten (World Scientific, Singapore 1985).

${ }^{3}$ G. W. Semenoff, Phys. Rev. Lett. 53, 2449 (1984).

${ }^{4}$ F. D. M. Haldane, Phys. Rev. Lett. 61, 2015 (1987).

${ }^{5}$ P. A. Lee, Phys. Rev. Lett. 71, 1887 (1993).

${ }^{6}$ A. A. Nersesyan, A. M. Tsvelik, and F. Wenger, Phys. Rev. Lett. 72, 2628 (1994).

${ }^{7}$ Y. Hasegawa, P. Lederer, T. M. Rice, and P. B. Wiegmann, Phys. Rev. Lett. 63, 907 (1989).

${ }^{8}$ X. G. Wen, F. Wilczek, and A. Zee, Phys. Rev. B 39, 11413 (1989).

${ }^{9}$ M. P. A. Fisher and E. Fradkin, Nucl. Phys. B 251, 457 (1984).

${ }^{10}$ K. Ohgushi, S. Murakami, and N. Nagaosa, Phys. Rev. B 62, R6065 (2000).

${ }^{11}$ C. L. Kane and E. J. Mele, Phys. Rev. Lett. 95, 146802 (2005).

${ }^{12}$ A. W. W. Ludwig, M. P. A. Fisher, R. Shankar, and G. Grinstein, Phys. Rev. B 50, 7526 (1993).

${ }^{13}$ Y. Hatsugai and M. Kohmoto, Phys. Rev. B 42, 8282 (1990).

${ }^{14}$ Landau levels in honeycomb lattice, including the $E=0$ level, were first obtained by J. W. McClure, Phys. Rev. 104, 666 (1956).

${ }^{15}$ Y. Zheng and T. Ando, Phys. Rev. B 65, 245420 (2002).

${ }^{16}$ V. P. Gusynin and S. G. Sharapov, Phys. Rev. Lett. 95, 146801 (2005).

${ }^{17}$ K. S. Novoselov, A. K. Geim, S. V. Morozov, D. Jiang, M. J. Kutsnelson, I. V. Grigovieva, S. V. Dubonos, and A. A. Firsov, Nat. Phys. 2, 177 (2006).

${ }^{18}$ N. M. R. Peres, F. Guinea, and A. H. Castro Neto, Phys. Rev. B 73, 125411 (2006).

${ }^{19}$ K. S. Novoselov, E. McCann, S. V. Morozov, V. I. Fal'ko, M. I. Katsnelson, V. Zeitler, D. Jiang, F. Schedin, and A. K. Geim, Nature (London) 438, 197 (2005).

${ }^{20}$ Y. Zhang, Y.-W. Tan, H. L. Stormer, and P. Kim, Nature (London) 438, 201 (2005).

${ }^{21}$ T. Ando, J. Phys. Soc. Jpn. 74, 777 (2005).

${ }^{22}$ M. Fujita, K. Wakabayashi, K. Nakada, and K. Kusakabe, J. Phys. Soc. Jpn. 65, 1920 (1996).

${ }^{23}$ K. Wakabayashi, M. Fujita, H. Ajiki, and M. Sigrist, Phys. Rev. B 59, 8271 (1999).

${ }^{24}$ M. V. Berry, Proc. R. Soc. London, Ser. A 392, 45 (1984).

${ }^{25}$ B. Simon, Phys. Rev. Lett. 51, 2167 (1983).

${ }^{26}$ X. G. Wen, Phys. Rev. B 40, 7387 (1989).

${ }^{27}$ Geometric Phases in Physics, edited by A. Shapere and F. Wilczek (World Scientific, Singapore, 1989).

${ }^{28}$ Y. Hatsugai, J. Phys. Soc. Jpn. 73, 2604 (2004).
${ }^{29}$ Y. Hatsugai, J. Phys. Soc. Jpn. 74, 1374 (2004).

${ }^{30}$ R. B. Laughlin, Phys. Rev. B 23, 5632 (1981).

${ }^{31}$ B. I. Halperin, Phys. Rev. B 25, 2185 (1982).

${ }^{32}$ Y. Hatsugai, Phys. Rev. B 48, 11851 (1993).

${ }^{33}$ Y. Hatsugai, Phys. Rev. Lett. 71, 3697 (1993).

${ }^{34}$ E. Witten, Phys. Lett. 117B, 324 (1982).

${ }^{35}$ A. J. Niemi and G. W. Semenoff, Phys. Rev. Lett. 51, 2077 (1983).

${ }^{36}$ S. Ryu and Y. Hatsugai, Phys. Rev. Lett. 89, 077002 (2002).

${ }^{37} \Delta$ delineates a circle centered at $C_{0}=1+e^{i k_{2}}$ with a radius $r$ $=\sqrt{1+2\left(t^{\prime} / t\right) \cos k_{2}+\left(t^{\prime} / t\right)^{2}}$ in the complex $\Delta$ plane when $k_{1}$ is varied from 0 to $2 \pi$. By examining $C_{0}$ and $r$, we can show that we have a Dirac (linear) dispersion when the circle goes through the origin, which occurs for $-3 \leq t^{\prime} / t \leq 1$.

${ }^{38}$ D. R. Hofstadter, Phys. Rev. B 14, 2239 (1976).

${ }^{39}$ S. Ryu and Y. Hatsugai, Physica C 388-389, 90 (2003).

${ }^{40}$ D. N. Sheng, L. Sheng, and Z. Y. Weng, Phys. Rev. B 73, 233406 (2006).

${ }^{41}$ Topological QHE numbers when the transfer energies along three directions in the honeycomb lattice are varied including the isotropic case have recently been discussed by Y. Hasegawa, R. Konno, H. Nakano, and M. Kohmoto, cond-mat/0604433; Y. Hasegawa and M. Kohmoto, cond-mat/0603345.

${ }^{42} \mathrm{~A}$ fermion doubling in honeycomb lattice in zero total flux was discussed in ${ }^{4}$.

${ }^{43}$ R. Rammal, J. Phys. (Paris) 46, 1345 (1985).

${ }^{44}$ D. J. Thouless, M. Kohmoto, M. P. Nightingale, and M. den Nijs, Phys. Rev. Lett. 49, 405 (1982).

${ }^{45}$ M. Kohmoto, Ann. Phys. (N.Y.) 160, 355 (1985).

${ }^{46}$ H. Aoki and T. Ando, Solid State Commun. 38, 1079 (1981).

${ }^{47}$ T. Fukui, Y. Hatsugai, and H. Suzuki, J. Phys. Soc. Jpn. 74, 1674 (2005).

${ }^{48}$ Y. Hatsugai, T. Fukui, and H. Suzuki, Physica E (Amsterdam) 34, 336 (2006).

${ }^{49}$ M. Lüscher, Commun. Math. Phys. 85, 39 (1982).

${ }^{50}$ A. Phillips, Ann. Phys. (N.Y.) 161, 399 (1985).

${ }^{51}$ A. Phillips and D. Stone, Commun. Math. Phys. 103, 599 (1986).

${ }^{52}$ A. Phillips and D. Stone, Commun. Math. Phys. 131, 255 (1990).

${ }^{53}$ M. Lüscher, Nucl. Phys. B 538, 515 (1999).

${ }^{54}$ T. Fujiwara, H. Suzui, and K. Wu, Prog. Theor. Phys. 105, 789 (2005).

${ }^{55}$ Y. Hatsugai, J. Phys.: Condens. Matter 9, 2507 (1997).

${ }^{56}$ K. Kusakabe and Y. Takagi, Mol. Cryst. Liq. Cryst. Sci. Technol., Sect. A 387, 7 (2002).

${ }^{57}$ H. Aoki, Surf. Sci. 263, 137 (1992).

${ }^{58}$ B. A. Bernevig, T. L. Hughes, S. C. Zhang H-D Chen, and C. Wu, cond-mat/0607774 (unpublished). 